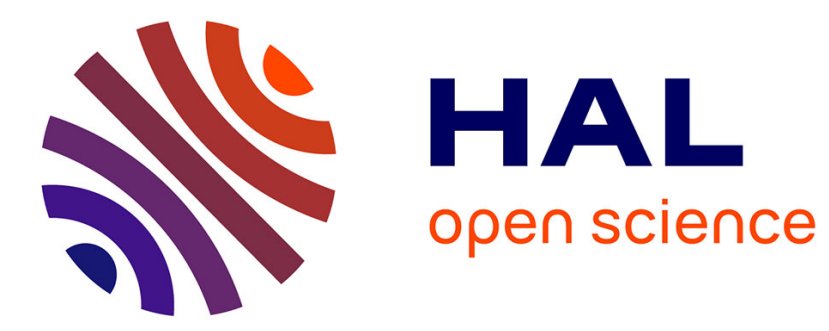

\title{
Fast Control Systems: Nonlinear Approach
}

Andrey Polyakov

\section{To cite this version:}

Andrey Polyakov. Fast Control Systems: Nonlinear Approach. New Perspectives and Applications of Modern Control Theory, Springer, pp.287-316, 2017, 10.1007/978-3-319-62464-8_12 . hal-01691572

\section{HAL Id: hal-01691572 \\ https://hal.inria.fr/hal-01691572}

Submitted on 24 Jan 2018

HAL is a multi-disciplinary open access archive for the deposit and dissemination of scientific research documents, whether they are published or not. The documents may come from teaching and research institutions in France or abroad, or from public or private research centers.
L'archive ouverte pluridisciplinaire HAL, est destinée au dépôt et à la diffusion de documents scientifiques de niveau recherche, publiés ou non, émanant des établissements d'enseignement et de recherche français ou étrangers, des laboratoires publics ou privés. 


\title{
Fast Control Systems: Nonlinear Approach
}

Andrey Polyakov

\begin{abstract}
This chapter treats the problem of fast control design for nonlinear systems. First, we discusses the question: which nonlinear system can be called fast? Next, we develop some tools for analysis and design of such control systems. The method generalized homogeneity is mainly utilized for these purposes. Finally, we survey possible research directions of the fast control systems.
\end{abstract}

\section{Introduction}

The Olympic motto "Citius, Altius, Fortius" ("Faster, Higher, Stronger") precisely reflects evolution trend of any known engineering invention. Each next generation of aircrafts (automobiles, trains, robots and so on) has to be faster than previous one. They also must demonstrate higher performance and stronger robustness. Any related renovation usually requires an update of automatic control system.

The control theory is an interdisciplinary branch of both mathematics and engineering sciences. Consequently, on the one hand, some engineering invention precedes some renovation of control design methodology. Indeed, digital controllers (computes in general) replaced analog devices implying appearance of new research fields such as sampled, hybrid, event-driven control systems. On the other hand, new ideas to control theory may come from pure mathematics. For example, sliding mode control principles [48] are essentially based on ideas of theory of differential equations with discontinuous right hand sides [18] introduced in 1960, but the calculus of variations (see, e.g. [40]) initiated in 17th century underlies the optimal control design [40].

The fundamental background of the whole modern mathematical control theory has been presented in the seminal work [27] of A.M. Lyapunov "The general problem of the stability of motion" published in 1892. Today quantitative characteristics

Andrey Polyakov

Inria Lille, 40 avenue Halley, 59650, Villenueve d'Ascq, France, e-mail: andrey.polyakov@inria.fr 
of stability ( Lyapunov exponents, Lyapunov functions, etc) specify performance of control systems (such as convergence rate, input-to state stability, etc). Exponential stability determines the convergence rate of stable linear Ordinary Differential Equations (ODEs), which are still the most popular models of control systems.

Any mathematical model is just an approximation of a real (physical) plant under some assumptions on its behavior. Frequently, linear approximation (model) is not appropriate if we deal with "fast" control system, since linearizion neglects some nonlinear dynamics, which imply fast transition. To define "fast" control this paper uses linear system as the reference point for comparison of convergence rate. Namely, a nonlinear system is said to be fast if it demonstrates transients motions faster than any linear one, i.e. convergence rate of nonlinear system is faster than any exponential. In [38] such systems were called hyper exponential.

\subsection{Motivating Example}

Here we present an example of nonlinear model of physical system in order to pick out nonlinearities which may invoke fast transitions.

Let us consider mechanical system consisting of a rigid body moving laterally on a contact surface and in some viscous environment (fluid). A simplest real-life example of such mechanical system is a car moving on a flat road with a sufficiently high velocity (more than $50 \mathrm{~km} / \mathrm{h}$ ).

Let $z$ be position of the center of mass of the body in an inertial frame. The equation describing motion of this system has the form

$$
\dot{z}(t)=v(t), \quad m \dot{v}(t)=F(t), \quad t>0, \quad z(t) \in \mathbb{R},
$$

where $v(t)$ is the velocity, $m$ is a mass of the body, but $F$ is sum of external forces.

We study only deceleration motion of this system assuming that at the initial instant of time the system has some non-zero velocity $\dot{z}(0)=v(0) \neq 0$. Dissipation of the energy may be caused by several external forces. To discover hyper exponential behavior it is sufficient to consider only two of them:

- drag force (fluid resistance) is proportional to the square of the velocity [17]

$$
F_{\text {drag }}(t)=-k_{\text {drag }} v^{2}(t) \operatorname{sign}[v(t)] \text {, }
$$

where $k_{d r a g}>0$ is the coefficient of fluid resistance and the sign function is

$$
\operatorname{sign}[\rho]=\left\{\begin{array}{cc}
1 & \text { if } \rho>0 \\
0 & \text { if } \rho=0 \\
-1 & \text { if } \rho<0
\end{array}\right.
$$

- $d r y$ friction force is nearly velocity independent and given by the next model [3]

$$
F_{d r y}(t)=-k_{d r y} \operatorname{sign}[v(t)]
$$

where $k_{d r y}>0$ is the coefficient of dry friction.

Usually the friction models also contain some linear terms (proportional to velocity). We skip them for simplicity of analysis, since they will not effect the final conclusions about convergence rate. 
The sum of external forces $F(t)$ can be represented as follows

$$
F(t)=F_{d r a g}(t)+F_{d r y}(t)=-\left(k_{d r y}+k_{d r a g} v^{2}(t)\right) \operatorname{sign}[v(t)] .
$$

and the differential equation describing evolution of the velocity of the body has the form:

$$
m \dot{v}(t)=-\left(k_{d r y}+k_{d r a g} v^{2}(t)\right) \operatorname{sign}[v(t)] .
$$

It is easy to see that $v=0$ is the equilibrium of the last equation, which is globally stable, $v(t) \rightarrow 0$ as $t \rightarrow+\infty$. The equation can be solved analytically:

$$
v(t)=\tan \left(\arctan (|v(0)|)-\frac{\sqrt{k_{d r y} k_{d r a g}}}{m} t\right) \operatorname{sign}[v(0)] .
$$

This immediately implies $v(t)=0$ for $t \geq \frac{m}{\sqrt{k_{\text {dry }} k_{\text {dra }}}} \arctan (|v(0)|)$. Since $\arctan$ is bounded function we conclude that independently of initial velocity the motion of the body terminates no later than

$$
T_{\max }=\frac{m \pi}{2 \sqrt{k_{d r y} k_{d r a g}}} .
$$

The deceleration rate of this mechanical system is hyper exponential since it faster than any exponential in the following sense:

$\forall C>0, \forall \alpha>0: \exists v(0) \in \mathbb{R}, \exists t^{\prime}>0$ such that $|v(t)|<C|v(0)| e^{-\alpha t}, \forall t>t^{\prime}$. Below we use this property for the rigorous definition of fast stability.

\subsection{State of the Art}

It seems that the first separation of fast and slow motions of dynamical systems have been systematically studied in the context of the so-called singularly perturbed ODEs [47], which contain a small parameter multiplied by the highest derivative. Tending this parameter to zero implies boosting system transitions in a certain subspace. In this paper we follow another philosophy.

According to the motivating example given above fast motions are caused by the nonlinearity of the plant. Dependently of the type of nonlinearity the fast transitions can be guaranteed locally or globally. Description of the behavior of such "fast nonlinear systems" can be efficiently embedded into Lyapunov Theory of stability. Some results in this context can be discovered in the literature. In particular, fast stability of ODEs is represented by the notions of finite-time and fixed-time stabilities [50], [43], [20], [11], [6], [29], [26], [2], [14], [33], [39], but hyper exponential transitions are studied in [38] as fast behavior of time delay systems. Fast models described by partial differential equations may demonstrate the so-called finite-time extinction property [46], [19], [31], [10] also known as super stability [5], [13]. This 
chapter surveys systematically all the mentioned concepts and presents some tools for analysis and design of fast (in particular fixed-time) control systems.

\section{Stability and Fast Convergence}

The concept of stability introduced by A.M. Lyapunov [27] considers a nominal motion $x_{t_{0}, x_{0}}^{*}(t), t \geq t_{0}$ of a dynamic system with initial state $x_{t_{0}, x_{0}}^{*}\left(t_{0}\right)=x_{0}$ and perturbed motions obtained for initial conditions $x_{0}+\delta$, where $\delta$ is a perturbation. If small perturbations of initial conditions imply small deviations of perturbed motions from $x_{t_{0}, x_{0}}^{*}(t)$ then the nominal motion is called stable. In this chapter we deal only with stability analysis of the zero solution (i.e. the origin), since making the change of variables $y=x-x^{*}$ we transform stability analysis problem to the latter case.

Let us consider the nonlinear system

$$
\begin{gathered}
\dot{x}(t)=f(t, x(t)), t>t_{0} \in \mathbb{R}, \\
x\left(t_{0}\right)=x_{0} \in \mathbb{R}^{n},
\end{gathered}
$$

where $f: \mathbb{R} \times \mathbb{R}^{n}$ may be a non-Lipschitz or even discontinuous function. In the latter case we assume that $f$ satisfies conditions of existence of Filippov solutions [18], which almost everywhere satisfy the differential inclusion $\dot{x}(t) \in F(t, x(t)), t>0$, where $F: \mathbb{R} \times \mathbb{R}^{n} \rightrightarrows \mathbb{R}^{n}$ is a set-valued function contracted from $f$ using a proper regularization procedure. Three the most popular regularization procedures are surveyed in [18], [39]. Note that in general the Cauchy problem (1)-(2) may have nonunique solutions implying two types of stability: weak stability (a stability property holds for some solution) and strong stability (a stability property holds for all solutions) (see, for example, [44], [43], [18]). Weak stability usually is not enough for robust control purposes. All conditions presented in definitions below are assumed to be held for all solutions of (1)-(2).

\subsection{Non-Rated Stability}

Assume that the origin is the equilibrium point of (1), i.e. $f(t, 0)=0$ (or $0 \in F(t, 0)$ ) for all $t \in \mathbb{R}$. This means that $x^{*}(t) \equiv 0$ is the solution to (1), (2) with $x_{0}=0$.

Definition 1 (Lyapunov stability). The origin of the system (1) is said to be Lyapunov stable if for $\forall \varepsilon \in \mathbb{R}_{+}$and $\forall t_{0} \in \mathbb{R}$ there exists $\delta=\delta\left(\varepsilon, t_{0}\right) \in \mathbb{R}_{+}$such that for $\forall x_{0} \in \mathbb{R}^{n}:\left\|x_{0}\right\|<\delta$ any solution $x_{t_{0}, x_{0}}(t)$ of Cauchy problem (1), (2) exists for $t>t_{0}$ and $\left\|x_{t_{0}, x_{0}}(t)\right\|<\varepsilon$ for $t>t_{0}$.

If the function $\delta$ does not depend on $t_{0}$ then the origin is called uniformly Lyapunov stable. If $f(t, x)$ is independent of $t$ (time-invariant) and the zero solution of (1) is Lyapunov stable, then it is uniformly Lyapunov stable. 
Proposition 1. If the origin of the system (1) is Lyapunov stable then $x(t)=0$ is the unique solution of Cauchy problem (1), (2) with $x_{0}=0$ and $t_{0} \in \mathbb{R}$.

The origin, which does not satisfy any condition from Definition 1, is unstable.

Definition 2 (Asymptotic stability). The origin of the system (1) is said to be asymptotically stable if it Lyapunov stable and if for any $t_{0} \in \mathbb{R}$ there exists an open set $U\left(t_{0}\right) \subseteq \mathbb{R}^{n}: 0 \in \operatorname{int}\left(U\left(t_{0}\right)\right)$ such that $\forall x_{0} \in U\left(t_{0}\right)$ one holds $\lim _{t \rightarrow+\infty}\left\|x_{t_{0}, x_{0}}(t)\right\|=0$.

The set $U\left(t_{0}\right)$ is called domain of attraction. It is always a neighborhood of the origin. If $U\left(t_{0}\right)=\mathbb{R}^{n}$ then the asymptotically stable origin of the system (1) is called globally asymptotically stable.

The uniform asymptotic stability asks for more strong attractivity property.

Definition 3 (Uniform asymptotic stability). The origin of the system (1) is said to be uniformly asymptotically stable if it is asymptotically stable with a time-invariant attraction domain $U \subseteq \mathbb{R}^{n}$ and $\forall R>0, \forall \varepsilon>0$ there exists $t^{*}=t^{*}(R, \varepsilon) \in \mathbb{R}_{+}$such that $\forall x_{0} \in U:\left\|x_{0}\right\|<R$ and $\forall t_{0} \in \mathbb{R}$ one holds $\left\|x_{t_{0}, x_{0}}(t)\right\|<\varepsilon$ for $t>t_{0}+t^{*}$.

If $U=\mathbb{R}^{n}$ then a uniformly asymptotically stable origin of the system (1) is called globally uniformly asymptotically stable. Uniform asymptotic stability always implies asymptotic stability. The converse holds for time-invariant systems.

Proposition 2 ([9], Proposition 2.2, page 78). Let a set-valued function $F: R^{n} \rightarrow$ $\mathbb{R}^{n}$ be defined and upper-semicontinuous in $\mathbb{R}^{n}$. Let $F(x)$ be nonempty, compact and convex for any $x \in \mathbb{R}^{n}$. If the origin of the system $\dot{x} \in F(x)$ is asymptotically stable then it is uniformly asymptotically stable.

\subsection{Rated Stability}

In order to provide good performance to control system a rate of transition processes has to be adjusted. An asymptotic stability does not characterize convergence rate, which should be somehow specified. The exponential stability is the classical example of the "rated" stability.

Definition 4 (Exponential stability). The origin of the system (1) is said to be exponentially stable if it is asymptotically stable and for any $t_{0} \in \mathbb{R}$ there exists an attraction domain $U\left(t_{0}\right)$ and $C=C\left(t_{0}\right)>0, r=r\left(t_{0}\right)>0$ such that

$$
\left\|x_{t_{0}, x_{0}}(t)\right\| \leq C\left\|x_{0}\right\| e^{-r\left(t-t_{0}\right)}, \quad t>t_{0}, \quad x_{0} \in U\left(t_{0}\right) .
$$

The exponential stability is uniform if $U\left(t_{0}\right), C\left(t_{0}\right)$ and $r\left(t_{0}\right)$ are time invariant. The parameter $r$ defines the rate of exponential convergence. Obviously, exponential stability implies both Lyapunov stability and asymptotic stability, and it is usually exploited by linear control theory. 
Definition 5 (Hyper Exponential Stability)). The origin of the system (1) is said to be hyper exponentially stable if it is exponentially stable with $U\left(t_{0}\right) \subseteq \mathbb{R}^{n}, t_{0} \in \mathbb{R}$ and

$$
\forall C>0, \forall r>0, \quad \exists x_{0} \in U\left(t_{0}\right), \exists t^{\prime}=t^{\prime}\left(t_{0}\right):\left\|x_{t_{0}, x_{0}}(t)\right\|<C\left\|x_{0}\right\| e^{-r\left(t-t_{0}\right)}, t>t_{0}+t^{\prime} .
$$

The origin is uniformly hyper exponentially stable if $U\left(t_{0}\right), t^{\prime}\left(t_{0}\right)$ are time-invariant. Definition 5 also introduces kind of "non-rated" stability since it does not provide any quantitative index to characterize (compare) hyper exponential convergence rates.

Given vector $\alpha=\left(\alpha_{0}, \alpha_{1}, \ldots, \alpha_{r}\right)^{\top} \in \mathbb{R}_{+}^{r+1}, \alpha_{i}>0$ with $r \geq 0$ let us define recursively the following family of functions

$$
\rho_{0, \alpha}(s)=\alpha_{0} s, \quad \rho_{i, \alpha}(s)=\alpha_{i}\left(e^{\rho_{i-1, \alpha}(s)}-e^{\rho_{i-1, \alpha}(0)}\right), \quad i=1,2, \ldots, r .
$$

Obviously $\rho_{i, \alpha}(0)=0$. The Fig. 1 depicts $e^{-\rho_{i, \alpha}(t)}, t>0$ for $i=1,2$ and $\alpha_{i}=1$ in a logarithmic scale in order to show the decay rate.

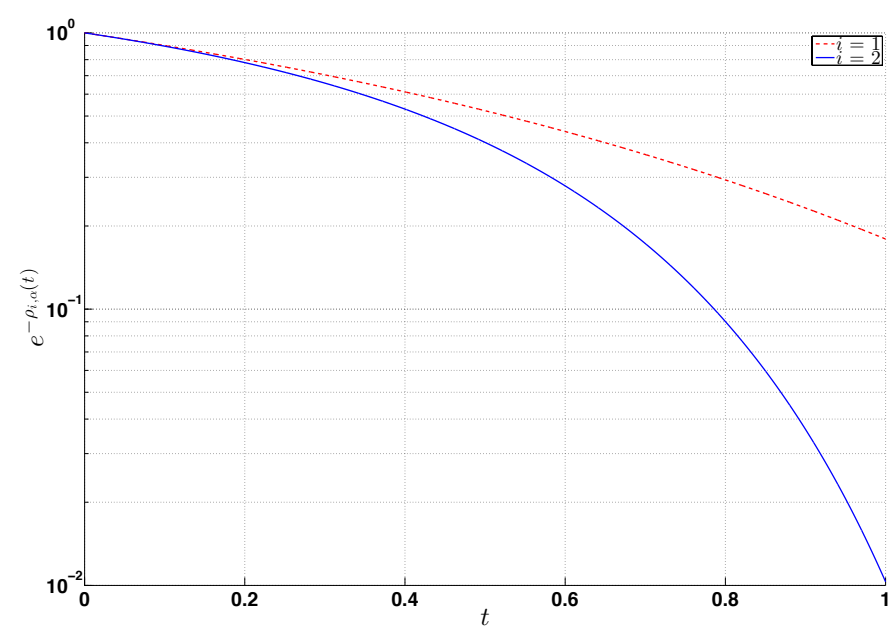

Fig. 1 Hyper exponential rate of convergence

Definition 6 (Rated Hyper Exponential Stability, [38]). The origin of the system (1) is said to be hyper exponentially stable of degree $r \geq 0$, if it is hyper exponentially stable with $U\left(t_{0}\right) \subseteq \mathbb{R}^{n}, t_{0} \in \mathbb{R}$ and $\exists C=C\left(t_{0}\right)>0, \exists \alpha=\alpha\left(t_{0}\right) \in \mathbb{R}_{+}^{r+1}$ such that

$$
\left\|x_{t_{0}, x_{0}}(t)\right\| \leq C\left\|x_{0}\right\| e^{\left.-\rho_{r, \alpha}\left(t-t_{0}\right)\right)}, \quad t>t_{0}, \quad x_{0} \in U\left(t_{0}\right) .
$$

The rated hyper exponential stability becomes uniform if $U\left(t_{0}\right), C\left(t_{0}\right)$ and $r\left(t_{0}\right)$ are time invariant. By analogy with the exponential case let us call the vector $\alpha$ by the rate of hyper exponential convergence. If the hyper exponential stability of degree $r$ is equal to zero then rate of convergence becomes exponential. 
Example 1. The right-hand side of the system

$$
\dot{x}(t)=-2 x(t)|\ln | x(t)||, \quad t>t_{0}, \quad x\left(t_{0}\right)=x_{0} \in U:=(-0.5,0.5),
$$

is continuous at the origin (and, in fact, in $\mathbb{R}$ ), which is locally asymptotically stable in the attraction domain $U$. The considered system has the following an explicit solution

$$
x(t)=x_{0} e^{\ln \left(\left|x_{0}\right|\right)\left(e^{2\left(t-t_{0}\right)}-1\right)} \quad \text { for } \quad x_{0} \in U .
$$

Since, obviously, we have $|x(t)| \leq\left|x_{0}\right| e^{-\ln (2)\left(e^{2\left(t-t_{0}\right)}-e^{2 \cdot 0}\right)}$ for all $x_{0} \in U$ then the origin of the consider system is uniformly hyper exponentially stable of degree 1 with the rate of hyper exponential convergence given by the vector $\alpha=(2, \ln 2)^{\top}$.

Remark 1. Similarly to the exponential case, the index $\alpha$ (rate of hyper exponential convergence) can be utilized for comparison of decay rates. Indeed, it is easy to show that for $\alpha, \beta \in \mathbb{R}_{+}^{r+1}$ the inequality $\alpha \geq \beta$ (understood in a component-wise sense) implies $e^{-\rho_{r, \alpha}(s)} \leq e^{-\rho_{r, \beta}(s)}$ for all $s \geq 0$.

\subsection{Non-asymptotic convergence}

The motivating example considered in Subsection 1.1 presents the mechanical system, which has non-asymptotic transitions, i.e. any trajectory reach the equilibrium after a finite instant of time.

Definition 7 (Finite-time stability ([43], [6])). The origin of the system (1) is said to be finite-time stable if it is Lyapunov stable in $U\left(t_{0}\right) \in \mathbb{R}^{n}, t_{0} \in \mathbb{R}$ and finite-time attractive : $\forall x_{0} \in U\left(t_{0}\right), \exists T=T\left(t_{0}, x_{0}\right) \geq 0$ such that $x_{t_{0}, x_{0}}(t)=0, \forall t \geq t_{0}+T$.

Finite-time transitions are required for many control applications. For example, antimissile control has to be designed only on a finite interval of time, since there is nothing to control after missile explosion.

Obviously that finite-time stability always implies asymptotic stability. The settling-time function $T$ of time-invariant finite-time stable system (1) is independent of $t_{0}$, i.e. $T=T\left(x_{0}\right)$. However, in contrast to asymptotic and Lyapunov stability, finite-time stability of a time-invariant system, in general, does not imply uniform finite-time stability, which asks at least for boundednees of the settling time function in a neighborhood of the origin.

Example 2 ([6], page 756). Let a vector field $f: \mathbb{R}^{2} \rightarrow \mathbb{R}^{2}$ of a time-invariant system be defined on the quadrants

$$
\begin{array}{ccc}
Q_{I}=\left\{x \in \mathbb{R}^{2} \backslash\{0\}: x_{1} \geq 0, x_{2} \geq 0\right\} & Q_{I I}=\left\{x \in \mathbb{R}^{2}: x_{1}<0, x_{2} \geq 0\right\} \\
Q_{I I I}=\left\{x \in \mathbb{R}^{2}: x_{1} \leq 0, x_{2}<0\right\} & Q_{I V}=\left\{x \in \mathbb{R}^{2}: x_{1}>0, x_{2}<0\right\}
\end{array}
$$

as shown in Fig. 2. The vector field $f$ is continuous, $f(0)=0$ and $x=\left(x_{1}, x_{2}\right)^{T}=$ $(r \cos (\theta), r \sin (\theta))^{T}, r>0, \theta \in[0,2 \pi)$. In [6] it was shown that this system is 


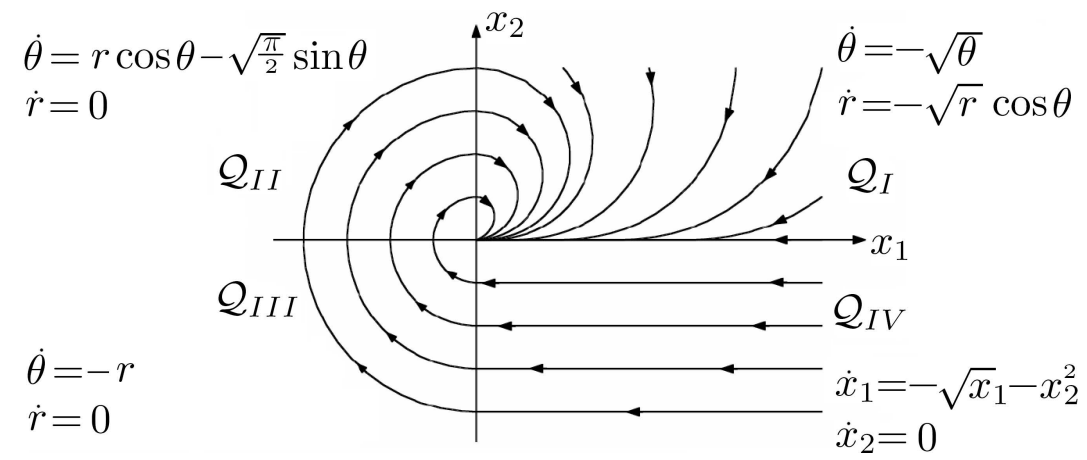

Fig. 2 Example of S.P. Bhat and D. Bernstein [6].

finite-time stable and, moreover, it is uniformly asymptotically stable. However, for the sequence of the initial conditions $x_{0}^{i}=(0,-1 / i)^{T}, i=1,2, \ldots$ we have (see [6] for the details) $x_{0}^{i} \rightarrow 0$ and $T\left(x_{0}^{i}\right) \rightarrow+\infty$ as $i \rightarrow+\infty$. So, considering an open ball $B(r)$ of the radius $r$ with the center at the origin we have for any $r>0$ that

$$
\sup _{x_{0} \in B(r)} T\left(x_{0}\right)=+\infty
$$

i.e. the trajectories of the considered system converge to zero in finite-time, but non-uniformly with respect to the initial conditions.

Definition 8 (Uniform finite-time stability, [29], [39]). The origin of the system (1) is said to be uniformly finite-time attractive if it is finite-time stable in a timeinvariant attraction domain $U \subseteq \mathbb{R}^{n}$ and the settling time function $T: \mathbb{R} \times U \rightarrow \mathbb{R}$ is locally bounded on $\mathbb{R} \times U$ uniformly on the first argument, i.e.

$$
\forall y \in U, \quad \exists \varepsilon>0 \quad \text { such that } \sup _{t_{0} \in \mathbb{R},\left\|x_{0}-y\right\|<\varepsilon} T\left(t_{0}, x_{0}\right)<+\infty .
$$

The mechanical system considered in the motivating example presented in Subsection 1.1 is obviously uniformly finite-time stable even if the drag force is assumed to be equal zero. The quadratic term provided by drag force implies more fast non-asymptotic transitions characterized by the next definition.

Definition 9 (Fixed-time stability, [33]). The origin of the system (1) is said to be fixed-time stable if it is uniformly finite-time stable in $U \subset \mathbb{R}^{n}$ and the settling time function $T\left(t_{0}, x_{0}\right)$ is bounded on $\mathbb{R} \times U$, i.e.

$$
\exists T_{\max }>0: x_{t_{0}, x_{0}}(t)=0, \quad t>t_{0}+T_{\max }, \quad \forall t_{0} \in \mathbb{R}, \quad \forall x_{0} \in U .
$$

If $U=\mathbb{R}^{n}$ then the origin of the system (1) is globally stable in the sense of definitions given above.

Obviously all finite-time and fixed-time stable systems are also hyper exponentially stable (locally or globally). 


\section{Mathematical Tools for Analysis of Fast Nonlinear Systems}

\subsection{Generalized Homogeneity}

Homogeneity widely studied in control theory [23], [41], [32], [15], [37] is a sort of symmetry of an object (e.g. function or vector field) with respect to some group of transformations called dilation.

\subsubsection{Dilation Group}

Let $\|\cdot\|$ be a norm in $\mathbb{R}^{n}$ and $\|\cdot\|_{\mathbb{A}}$ be the matrix norm induced by $\|\cdot\|$, i.e. $\|A\|_{\mathbb{A}}=$ $\sup _{u \in \mathbb{R}^{n}} \frac{\|A u\|}{\|u\|}$ if $A \in \mathbb{R}^{n \times n}$.

Definition 10. A map $\mathfrak{d}: \mathbb{R} \rightarrow \mathbb{R}^{n \times n}$ is called dilation in $\mathbb{R}^{n}$ if it satisfies

- the group property: $\mathfrak{d}(0)=I \in \mathbb{R}^{n \times n}$ and $\mathfrak{d}(t+s)=\mathfrak{d}(t) \mathfrak{d}(s)$ for $t, s \in \mathbb{R}$;

- the continuity property: the map $\mathfrak{d}$ is continuous in the norm $\|\cdot\|_{\mathbb{A}}$, i.e.

$\forall t>0, \forall \varepsilon>0, \quad \exists \delta=\delta(t, \varepsilon)>0:|s-t|<\delta \Rightarrow\|\mathfrak{d}(s)-\mathfrak{d}(t)\|_{\mathbb{A}} \leq \varepsilon$.

- the limit property: $\lim _{s \rightarrow-\infty}\|\mathfrak{d}(s) u\|=0$ and $\lim _{s \rightarrow+\infty}\|\mathfrak{d}(s) u\|=+\infty$ uniformly on $u \in S$, where $S=\left\{u \in \mathbb{R}^{n}:\|u\|=1\right\}$ is the unit sphere in $\mathbb{R}^{n}$.

The dilation given by this definition was originally introduced in [35] for abstract Banach spaces $\mathbf{B}$ in the form a strongly continuous group of linear bounded operators [30], i.e. $\mathfrak{d}(s) \in \mathscr{L}(\mathbf{B}, \mathbf{B})$ and $\mathfrak{d}(\cdot) u: \mathbb{R} \rightarrow \mathbf{B}$ is continuous for any $u \in \mathbf{B}$. In a finite dimensional space any strongly continuous group is uniformly continuous, i.e. continuous in the norm of the Banach space $\mathscr{L}(\mathbf{B}, \mathbf{B})$ - the space of linear bounded operators $\mathbf{B} \rightarrow \mathbf{B}$. For $\mathbf{B}=\mathbb{R}^{n}$ we derive $\mathscr{L}(\mathbf{B}, \mathbf{B})=\mathbb{R}^{n \times n}$ and the definition 10 is equivalent Definition 1 from [35].

Example 3. The well-known dilations in $\mathbb{R}^{n}$

- uniform dilation (L. Euler 17th century) : $\mathfrak{d}(s)=e^{s}, s \in \mathbb{R}$;

- weighted dilation (Zubov 1958, [49]): $\mathfrak{d}(s)=\left(\begin{array}{cccc}e^{r_{1} s} & 0 & \ldots & 0 \\ 0 & e^{r_{2} s} & \ldots & 0 \\ \ldots & \ldots & \ldots & \ldots \\ 0 & 0 & \ldots & e^{r_{n}} s\end{array}\right), s \in \mathbb{R}, r_{i}>0$;

obviously, satisfy Definition 10. The geometric dilation [24], [23], [42] is more general since it allows the map $\mathfrak{d}(s): \mathbb{R}^{n} \rightarrow \mathbb{R}^{n}(s \in \mathbb{R})$ to be non-linear.

The matrix $G_{\mathfrak{d}} \in \mathbb{R}^{n \times n}$ defined as $G_{\mathfrak{d}}=\lim _{s \rightarrow 0} \frac{\mathfrak{d}(s)-I}{s}$ is known (see, e.g. [30, Ch. 1]) as the generator of the group $\mathfrak{d}(s)$. It satisfies the following properties

$$
\frac{d}{d s} \mathfrak{d}(s)=G_{\mathfrak{d}} \mathfrak{d}(s)=\mathfrak{d}(s) G_{\mathfrak{d}}, s \in \mathbb{R} \quad \text { and } \quad \mathfrak{d}(s)=e^{G_{\mathfrak{d}} s}=\sum_{i=0}^{+\infty} \frac{s^{i} G_{\mathfrak{d}}^{i}}{i !} .
$$

If we denote $\lfloor A\rfloor_{\mathbb{A}}=\inf _{u \in \mathbb{R}^{n}} \frac{\|A u\|}{\|u\|}, A \in \mathbb{R}^{n \times n}$ then the limit property implies that

$$
\begin{array}{ll}
\bullet \mathfrak{d}(s) \neq I \text { if } s \neq 0 ; & \bullet\lfloor\mathfrak{d}(s)\rfloor_{\mathbb{A}} \rightarrow+\infty \text { as } s \rightarrow+\infty \\
\bullet\|\mathfrak{d}(s)\|_{\mathbb{A}} \rightarrow 0 \text { as } s \rightarrow-\infty ; & \left.\bullet\left\lfloor G_{\mathfrak{d}}\right\rfloor>0 \text { (i.e. } \operatorname{ker} G_{\mathfrak{d}}=\{\boldsymbol{0}\}\right)
\end{array}
$$


Definition 11. The dilation $\mathfrak{d}$ is monotone on $\mathbb{R}^{n}$ if $\|\mathfrak{d}(s)\|_{\mathbb{A}}<1$ for $s<0$.

Monotonicity of the dilation depends on the norm $\|\cdot\|$.

Example 4. The dilation $\mathfrak{d}(s)=e^{s}\left(\begin{array}{cc}\cos (s) & \sin (s) \\ -\sin (s) & \cos (s)\end{array}\right)$ with $G_{\mathfrak{d}}=\left(\begin{array}{cc}1 & 1 \\ -1 & 1\end{array}\right)$ is monotone on $\mathbb{R}^{2}$ equipped with weighted norm $\|u\|_{P}=\sqrt{u^{\top} P u}$ if $P=\left(\begin{array}{cc}1 & 1 / \sqrt{2} \\ 1 / \sqrt{2} & 1\end{array}\right)>0$ and it is non-monotone if, for example, $P=\left(\begin{array}{cc}1 & 3 / 4 \\ 3 / 4 & 1\end{array}\right)>0$. In the latter case, the curve $\{\mathfrak{d}(s) u: s \in \mathbb{R}\}$ may cross the unit sphere in two different points.

Theorem 1. A dilation $\mathfrak{d}$ is monotone on $\mathbb{R}^{n}$ if and only if one of the conditions holds

1) $\lfloor\mathfrak{d}(s)\rfloor_{\mathbb{A}}>1$ for $s>0$;

2) the continuous function $\|\mathfrak{d}(\cdot) u\|: \mathbb{R} \rightarrow \mathbb{R}_{+}$is strictly increasing for any $u \in S$, where $S:=\left\{u \in \mathbb{R}^{n}:\|u\|=1\right\}$ is the unit sphere;

3) for any $u \in \mathbb{R}^{n}$ there exists a unique pair $\left(s_{0}, u_{0}\right) \in \mathbb{R} \times S$ such that $u=\mathfrak{d}\left(s_{0}\right) u_{0}$.

Proof. 1) For any $u \in S$ we have $1=\|u\|=\|\mathfrak{d}(s) \mathfrak{d}(-s) u\| \leq\|\mathfrak{d}(s)\|_{\mathbb{A}}\|\mathfrak{d}(-s) u\|$. Hence, $1 \leq\|\mathfrak{d}(s)\|_{\mathbb{A}}\lfloor\mathfrak{d}(-s)\rfloor_{\mathbb{A}}$ for any $s \in \mathbb{R}$.

2) On the one hand, $\operatorname{since} \sup _{u \in S}\|\mathfrak{d}(s) u\|=\left\|\mathfrak{d}(s) u_{s}\right\|$ for some $u_{s} \in S$ then strict monotonicity of $\|\mathfrak{d}(\cdot) u\|$ for any $u \in S$ implies $\|\mathfrak{d}(s)\|<1$ for $s<0$. On the other hand, if $\mathfrak{d}$ is monotone then for $u \neq 0$ and $s_{1}<s_{2}$ one has $\left\|\mathfrak{d}\left(s_{1}\right) u\right\|-\left\|\mathfrak{d}\left(s_{2}\right) u\right\|=$ $\left\|\mathfrak{d}\left(s_{1}\right) u\right\|-\left\|\mathfrak{d}\left(s_{2}-s_{1}\right) \mathfrak{d}\left(s_{1}\right) u\right\| \leq\left(1-\left\lfloor\mathfrak{d}\left(s_{2}-s_{1}\right)\right\rfloor_{\mathbb{A}}\right)\left\|\mathfrak{d}\left(s_{1}\right) u\right\|<0$. This implies the function $\|\mathfrak{d}(\cdot) u\|$ is strictly increasing for any $u \in S$.

3) Necessity. Existence and uniqueness of the pair $\left(s_{0}, u_{0}\right)$ such that $u_{0}=\mathfrak{d}\left(s_{0}\right) u \in$ $S$ for $u \in \mathbb{R}^{n}$ immediately follows continuity of the dilation and the condition 2). Sufficiency. If $u \in S$ is an arbitrary vector from the unit sphere then $\mathfrak{d}(s) u \notin S$ for all $s \neq 0$. Indeed, otherwise the pair $\left(s_{0}, u_{0}\right) \in \mathbb{R} \times S: u_{0}=\mathfrak{d}(s) u \in S$ is not unique. Hence, the limit property of the dilation (see, Definition 10) implies $\|\mathfrak{d}(s) u\|<1$ for all $s<0$ and all $u \in S$, i.e. $\|\mathfrak{d}(s)\|_{\mathbb{A}}<1$ for $s<0$.

Theorem 1 guarantees the functions $\|\mathfrak{d}(\cdot)\|_{\mathbb{A}}: \mathbb{R} \rightarrow \mathbb{R}_{+}$and $\lfloor\mathfrak{d}(\cdot)\rfloor_{\mathbb{A}}: \mathbb{R} \rightarrow \mathbb{R}_{+}$are also continuous and strictly increasing. Moreover, if $\|\cdot\|$ is $C^{k}$ outside the origin then the identity $\frac{d}{d s} \mathfrak{d}(s)=G_{\mathfrak{d}} \mathfrak{d}(s)$ guarantees that these functions are also $C^{k}$.

Definition 12. The dilation $\mathfrak{d}$ is said to be strictly monotone on $\mathbb{R}^{n}$ if there exists $\beta>0$ such that $\|\mathfrak{d}(s)\|_{\mathbb{A}} \leq e^{\beta s}$ for $s \leq 0$.

The dilation $\mathfrak{d}$ considered in Example 4 is strictly monotone on $\mathbb{R}^{2}$ equipped with the conventional Euclidian norm.

Theorem 2. Let $\mathfrak{d}$ be a dilation in $\mathbb{R}^{n}$ then

- the matrix $-G_{\mathfrak{d}}$ is Hurwitz, i.e. all eigenvalues $\lambda_{i}$ of $G_{\mathfrak{d}}$ are placed in the right complex half-plane; 
- for any $\beta \in\left(0, \beta^{*}\right]$ there exists a symmetric matrix $P \in \mathbb{R}^{n \times n}, P=P^{\top}$ such that

$$
P G_{\mathfrak{d}}+G_{\mathfrak{d}}^{\top} P \geq 2 \beta P, \quad P>0
$$

where $-\beta^{*}<0$ is the spectral abscissa of $-G_{\mathfrak{d}}$, i.e. $\beta^{*}=\min \Re\left(\lambda_{i}\right)$

- the dilation $\mathfrak{d}$ is strictly monotone with respect to the weighted Euclidean norm $\|\cdot\|=\sqrt{\langle\cdot, \cdot\rangle}$ induced by the inner product $\langle u, v\rangle=u^{\top} P v$ with $P$ satisfying (6) and

$$
\begin{gathered}
e^{\alpha s} \leq\lfloor\mathfrak{d}(s)\rfloor_{\mathbb{A}} \leq\|\mathfrak{d}(s)\|_{\mathbb{A}} \leq e^{\beta s} \text { if } s \leq 0, \quad e^{\beta s} \leq\lfloor\mathfrak{d}(s)\rfloor_{\mathbb{A}} \leq\|\mathfrak{d}(s)\|_{\mathbb{A}} \leq e^{\alpha s} \text { if } s \geq 0, \\
\alpha:=\sup _{z \in S}\left\langle G_{\mathfrak{d}} z, z\right\rangle=\frac{1}{2} \lambda_{\max }\left(P^{1 / 2} G_{\mathfrak{d}} P^{-1 / 2}+P^{-1 / 2} G_{\mathfrak{d}}^{\top} P^{1 / 2}\right)>0, \\
\beta:=\inf _{z \in S}\left\langle G_{\mathfrak{d}} z, z\right\rangle=\frac{1}{2} \lambda_{\min }\left(P^{1 / 2} G_{\mathfrak{d}} P^{-1 / 2}+P^{-1 / 2} G_{\mathfrak{d}}^{\top} P^{1 / 2}\right)>0 .
\end{gathered}
$$

Proof. Since $\frac{d}{d s} \mathfrak{d}(s)=G_{\mathfrak{d}} \mathfrak{d}(s), \mathfrak{d}(0)=I$ then $\mathfrak{d}(s)$ is the fundamental matrix of the linear system ODEs with the matrix $G_{\mathfrak{0}}$. The limit property of the dilation implies that this system of ODEs is globally asymptotically stable in the inverse time, i.e. the matrix $-G_{\mathfrak{d}}$ is Hurwitz. Hence, there exists a symmetric positive definite matrix such that (6) holds and for any $u \in S$ one has $\frac{d}{d s}\|\mathfrak{d}(s) u\|^{2}=u^{\top} \mathfrak{d}(s)^{\top}\left(G_{\mathfrak{d}}^{\top} P+\right.$ $\left.P G_{\mathfrak{d}}\right) \mathfrak{d}(s) u \geq 2 \beta\|\mathfrak{d}(s) u\|^{2}$. Similarly we derive $\frac{d}{d s}\|\mathfrak{d}(s) u\|^{2} \leq \alpha\|\mathfrak{d}(s) u\|^{2}$, i.e. the inequalities (7) hold.

Therefore, any dilation $\mathfrak{d}$ is strictly monotone on $\mathbb{R}^{n}$ equipped with the weighted Euclidian norm $\|u\|=\sqrt{u^{\top} P u}$ if the matrix $P>0$ satisfies (6).

\subsubsection{Homogeneous Norm}

The "homogeneous norm" is not a norm in the classical sense, since, in particular, the triangle inequality may not hold. However, it introduces a topology in $\mathbb{R}^{n}$ :

$$
S_{\mathfrak{d}}(r)=\left\{u \in \mathbb{R}^{n}:\|u\|_{\mathfrak{o}}=r\right\} \quad \text { and } \quad B_{\mathfrak{d}}(r)=\left\{u \in \mathrm{d}:\|u\|_{\mathfrak{o}}<r\right\}, \quad r>0,
$$

where $S_{\mathfrak{d}}(r)$ is the homogeneous sphere of the radius $r, B_{\mathfrak{d}}(r)$ is the homogeneous ball of the radius $r$ and $\|\cdot\|_{\mathfrak{d}}: \mathbb{R}^{n} \rightarrow \mathbb{R}$ is a function introduced by the next definition.

Definition 13. A continuous function $\|\cdot\|_{\mathfrak{o}}: \mathbb{R}^{n} \rightarrow \mathbb{R}_{+}$is said to be $\mathfrak{d}$-homogeneous norm if $\|u\|_{\mathfrak{o}} \rightarrow 0$ as $u \rightarrow \boldsymbol{0}$ and $\|\mathfrak{d}(s) u\|_{\mathfrak{o}}=e^{s}\|u\|_{\mathfrak{o}}>0$ for $u \in \mathbb{R}^{n} \backslash\{\boldsymbol{0}\}$ and $s \in \mathbb{R}$.

There are many ways to construct a homogeneous norm in $\mathbb{R}^{n}$ (see, e.g. [22], [41],[23], [35]). For monotone dilations we can introduce the canonical homogeneous norm as follows:

$$
\|u\|_{\mathfrak{d}}=e^{s_{u}} \quad: \quad\left\|\mathfrak{d}\left(-s_{u}\right) u\right\|=1 .
$$

In [34] this homogeneous norm was called canonical since it is induced by the canonical norm $\|\cdot\|$ in $\mathbb{R}^{n}$ and $\|x\|_{\mathfrak{o}}=\|x\|=1$ on the unit sphere $S$. Obviously 
that

$$
\left\lfloor\mathfrak{d}\left(\ln \|u\|_{\mathfrak{d}}\right)\right\rfloor_{\mathbb{A}} \leq\|u\| \leq\left\|\mathfrak{d}\left(\ln \|u\|_{\mathfrak{d}}\right)\right\|_{\mathbb{A}}
$$

where $\lfloor\mathfrak{d}(\cdot)\rfloor_{\mathbb{A}}$ and $\|\mathfrak{d}(\cdot)\|_{\mathbb{A}}$ are continuous and strictly increasing functions (see, Theorem 1).

In the view of Theorem 2 the symbol $\|\cdot\|_{\mathfrak{d}}$ denotes the canonical homogeneous norm by default.

Proposition 3. If $\mathfrak{d}$ is strictly monotone on $\mathbb{R}^{n}$ with dilation rate $\beta>0:\|\mathfrak{d}(s)\|_{\mathbb{A}} \leq$ $e^{\beta s}$ for $s<0$ (see Definition 12) then

- $\left|\left\|u_{1}\right\|_{\mathfrak{d}}^{\beta}-\left\|u_{2}\right\|_{\mathfrak{d}}^{\beta}\right| \leq\left\|u_{1}-u_{2}\right\|$ for $u_{1}, u_{2} \in \mathbb{R}^{n} \backslash B_{\mathfrak{d}}(1)$,

- the homogeneous norm $\|\cdot\|_{\mathfrak{d}}$ is Lipschitz continuous outside the origin;

- if the norm $\|\cdot\|$ is smooth outside the origin then the homogeneous norm $\|\cdot\|_{\mathfrak{o}}$ is also smooth outside the origin, $\frac{d}{d s}\|\mathfrak{d}(-s) u\|<0$ for $s \in \mathbb{R}, u \in \mathbb{R}^{n} \backslash\{\boldsymbol{0}\}$ and

$$
\frac{\partial\|u\|_{\mathfrak{o}}}{\partial u}=\left.\|u\|_{\mathfrak{d}} \frac{\left.\frac{\partial\|z\|}{\partial z}\right|_{z=\mathfrak{d}(-s) u}}{\left.\frac{\partial\|z\| \|}{\partial z}\right|_{z=\mathfrak{d}(-s) u} G_{\mathfrak{d}} \mathfrak{d}(-s) u}\right|_{s=\ln \|u\|_{\mathfrak{d}}} \quad \text { for } \quad u \in \mathbb{R}^{n} \backslash\{\boldsymbol{0}\}
$$

Proof.

- Since for $u_{i} \in \mathbb{R}^{n}$ we have $\left\|u_{i}\right\|_{\mathfrak{d}}=e^{s_{i}}:\left\|\mathfrak{d}\left(-s_{i}\right) u\right\|=1$ then $1=\left\|\mathfrak{d}\left(-s_{1}\right) u_{1}\right\|=$ $\left\|\mathfrak{d}\left(-s_{1}\right)\left(u_{1}-u_{2}\right)+\mathfrak{d}\left(s_{2}-s_{1}\right) \mathfrak{d}\left(-s_{2}\right) u_{2}\right\| \leq\left\|\mathfrak{d}\left(-s_{1}\right)\right\|_{\mathbb{A}}\left\|\left(u_{1}-u_{2}\right)\right\|+\| \mathfrak{d}\left(s_{2}-\right.$ $\left.s_{1}\right) \|_{\mathbb{A} .}$. For $1<\left\|u_{2}\right\|_{\mathfrak{d}}<\left\|u_{1}\right\|_{\mathfrak{d}}$ we have $0<s_{2}<s_{1}$ and $1 \leq e^{-\beta s_{1}}\left\|u_{1}-u_{2}\right\|+$ $e^{\beta s_{2}-\beta s_{1}}$ or equivalently, $\left\|u_{1}\right\|_{\mathfrak{d}}-\left\|u_{2}\right\|_{\mathfrak{o}} \leq\left\|u_{1}-u_{2}\right\|$.

- Lipschitz continuity follows from the proven inequality, the identity $\|\mathfrak{d}(s) u\|_{\mathfrak{o}}=$ $e^{s}\|u\|_{\mathfrak{d}}$ and monotonicity of the dilation.

- The existence of the unique function $s: \mathbb{R}^{n} \rightarrow \mathbb{R}$ such that $\|\mathfrak{d}(-s(u)) u\|=1$ has been proven in Theorem 1. Since the dilation is strictly monotone then $\frac{d}{d s}\|\mathfrak{d}(-s) u\|<0$ on $S$ (and, on $\left.\mathbb{R}^{n} \backslash\{0\}\right)$ for all $s \in \mathbb{R}$ (see, Theorem 1). Since the norm $\|\cdot\|$ is smooth them $\frac{d}{d s}\|\mathfrak{d}(-s) u\|=-\left.\frac{\partial\|z\|}{\partial z}\right|_{z=\mathfrak{d}(-s) u} G_{\mathfrak{d}} \mathfrak{d}(-s) u$. Taking into account $\frac{\partial}{\partial u}\|u\|_{\mathfrak{o}}=\left.e^{s} \frac{\partial s}{\partial u}\right|_{s=\ln \|u\|_{\mathfrak{o}}}$ the formula (9) can be derived using Implicit Function Theorem [12] applied to the equality $\|\mathfrak{d}(-s) u\|=1$.

\subsubsection{Homogeneous Functions and Homogeneous Vectors Fields}

Vector fields, which are symmetric in certain sense with respect to dilation $\mathfrak{d}$, have a lot of properties useful for control design and state estimation of both linear and nonlinear plants as well as for analysis of the convergence rate. 
Definition 14 (Homogeneous vector field (function) [35]). A vector field $f: \mathbb{R}^{n} \rightarrow$ $\mathbb{R}^{n}$ (a function $h: \mathbb{R}^{n} \rightarrow \mathbb{R}$ ) is said to be $\mathfrak{d}$-homogeneous of degree $v \in \mathbb{R}$ if

$$
\begin{array}{ccc}
f(\mathfrak{d}(s) u)=e^{v s} \mathfrak{d}(s) f(u), & \forall u \in \mathbb{R}^{n} \backslash\{\boldsymbol{0}\}, & \forall s \in \mathbb{R} . \\
\text { (resp. } h(\mathfrak{d}(s) u)=e^{v s} h(u), & \forall u \in \mathbb{R}^{n} \backslash\{\boldsymbol{0}\}, & \forall s \in \mathbb{R} .)
\end{array}
$$

Example 5. Let us consider the dilation $\mathfrak{d}(s)=e^{s}\left(\begin{array}{ccc}1 & 0 & 0 \\ 0 & \cos (s) & \sin (s) \\ 0 & -\cos (s) & \sin (s)\end{array}\right)$ that is strictly monotone with respect to the Euclidean norm $\|x\|=\sqrt{x^{T} x}$ and $G_{\mathfrak{d}}=\left(\begin{array}{ccc}1 & 0 & 0 \\ 0 & 1 & 1 \\ 0 & -1 & 1\end{array}\right)$. The vector field $f: \mathbb{R}^{3} \rightarrow \mathbb{R}^{3}$ defined as $f(x)=\left(\begin{array}{c}x_{2}^{2}+x_{3}^{2} \\ x_{1}^{2}\left(\cos \left(\ln \left|x_{1}\right|\right)+\sin \left(\ln \left|x_{1}\right|\right)\right) \\ x_{1}^{2}\left(\cos \left(\ln \left|x_{1}\right|\right)-\sin \left(\ln \left|x_{1}\right|\right)\right)\end{array}\right)$ and the function $h: \mathbb{R}^{3} \rightarrow \mathbb{R}$ given by $h=x_{1}^{3}+\left(x_{2}^{2}+x_{3}^{2}\right)^{\frac{3}{2}}$ are $\mathfrak{d}$-homogeneous of degree 1 and 3 , respectively.

Example 6 (Vector Field with Both Negative and Positive Homogeneity Degrees). Note that the vector field may have different degrees of homogeneity dependently of dilation group. Indeed, the linear vector field $f=A x: \mathbb{R}^{n} \rightarrow \mathbb{R}^{n}$ defined by a chain of integrators $A=\left(\begin{array}{cc}0 & I_{n-1} \\ 0 & 0\end{array}\right) \in \mathbb{R}^{n \times n}$ is $\mathfrak{d}_{1}$-homogeneous of degree 1 with $\mathfrak{d}_{1}(s)=$ $\operatorname{diag}\left\{e^{i s}\right\}_{i=1}^{n}$ and $\mathfrak{d}_{2}$-homogeneous of degree -1 with $\mathfrak{d}_{2}(s)=\operatorname{diag}\left\{e^{(n-i+1) s}\right\}_{i=1}^{n}$. Similar conclusion can be made for the chain of power integrators.

The homogeneity allows local properties (e.g. smoothness) of vector fields (functions) to be extended globally.

Corollary 1. Let the vector field $f: \mathbb{R}^{n} \rightarrow \mathbb{R}^{n}$ (a function $h: \mathbb{R}^{n} \rightarrow \mathbb{R}$ ) be $\mathfrak{d}$ homogeneous of degree $v \in \mathbb{R}$ and the norm $\|\cdot\|$ in $\mathbb{R}^{n}$ be defined according to Theorem 2 with $\beta=\beta^{*}$ and $\alpha \geq \beta$ given by (7).

i) If the function $h$ is bounded on the unit sphere $S$ then

a) for $v>0$ it is continuous at the origin, $h(\boldsymbol{0})=0$ and radially unbounded ${ }^{1}$ provided that $h(x) \neq 0$ on $S$;

b) for $v=0$ it is globally bounded in $\mathbb{R}^{n}$ and continuity of $h$ at the origin implies that $h=$ const;

c) for $v<0$ it is discontinuous at the origin, unbounded in any neighbourhood of the origin and $|h(x)| \rightarrow 0$ as $x \rightarrow \infty$;

ii) If the vector field $f$ is bounded on the unit sphere $S$ then

a) for $v+\beta>0$ it is continuous at the origin, $f(\boldsymbol{0})=0$ and radially unbounded if $f(x) \neq 0$ on $S$;

- for $v+\beta=0($ resp. $v+\alpha=0)$ it is bounded on $B_{\mathfrak{d}}(r)\left(\right.$ resp. on $\left.\mathbb{R}^{n} \backslash B_{\mathfrak{d}}(r)\right)$ for any fixed $r>0$;

${ }^{1}$ The vector field $f$ (resp. function $f$ ) is radially unbounded if $x \rightarrow \infty$ implies $\|f(x)\| \rightarrow+\infty$ (resp. $|h(x)| \rightarrow+\infty)$. 
- for $v+\beta=v+\alpha=0$ it is globally bounded on $\mathbb{R}^{n}$;

- for $v+\beta<0$ it is discontinuous at the origin, unbounded in any neighbourhood of the origin and $\|f(x)\| \rightarrow 0$ as $x \rightarrow \infty$;

iii) The vector field $f$ (resp. function $h$ ) is Lipschitz continuous on $\mathbb{R}^{n} \backslash\{\boldsymbol{0}\}$ if and only if it satisfies Lipschitz condition on $S$.

iv) If $h: \mathbb{R}^{n} \rightarrow \mathbb{R}$ is a $\mathfrak{d}$-homogeneous function of degree $v$ and differentiable on $S$ then it differentiable on $\mathbb{R}^{n} \backslash\{\boldsymbol{0}\}$ and

$$
\frac{\partial h(u)}{\partial u} G_{\mathfrak{d}} u=v h(u) \quad \text { for } \quad u \in \mathbb{R}^{n} \backslash\{\boldsymbol{0}\} .
$$

Proof. i) Since $h(u)=h\left(\mathfrak{d}\left(\ln \|u\|_{\mathfrak{o}}\right) z\right)=\|u\|_{\mathfrak{d}}^{v} h(z)$ where $z=\mathfrak{d}(-\ln \|u\|) u \in S$ and the homogeneous norm is continuous then $h$ is continuous at the origin if $v>0$, discontinuous at the origin if $v<0$ and globally bounded if $v=0$.

ii) Similarly for the vector field $f$ we derive $f(u)=\|u\|_{\mathfrak{d}}^{v} \mathfrak{d}\left(\ln \|u\|_{\mathfrak{d}}\right) f(z)$ and $\|u\|_{\mathfrak{o}}^{v}\left\lfloor\mathfrak{d}\left(\ln \|u\|_{\mathfrak{o}}\right)\right\rfloor_{\mathbb{A}}\|f(z)\| \leq\|f(u)\| \leq\|u\|_{\mathfrak{d}}^{V}\left\|\mathfrak{d}\left(\ln \|u\|_{\mathfrak{d}}\right)\right\|_{\mathbb{A}}\|f(z)\|$. For $\|u\|<1$ we have $\|u\|_{\mathfrak{d}}^{v+\alpha}\|f(z)\| \leq\|f(u)\| \leq\|u\|_{\mathfrak{d}}^{v+\beta}\|f(z)\|$ and $\|u\|_{\mathfrak{d}}^{v+\beta}\|f(z)\| \leq\|f(u)\| \leq$ $\|u\|_{\mathfrak{o}}^{v+\alpha}\|f(z)\|$ if $\|u\|>1$.

iii) Sufficiency. Let $u_{i} \in \mathbb{R}^{n} \backslash\{\mathbf{0}\}, i=1,2$ then $u_{i}=\mathfrak{d}\left(\ln \left\|u_{i}\right\|_{\mathfrak{o}}\right) z_{i}$ for some $z_{i} \in S$, $f\left(u_{1}\right)-f\left(u_{2}\right)=f\left(\mathfrak{d}\left(\ln \left\|u_{1}\right\|_{\mathfrak{o}}\right) z_{1}\right)-f\left(\mathfrak{d}\left(\ln \left\|u_{2}\right\|_{\mathfrak{o}}\right) z_{2}\right)=\left\|u_{1}\right\|_{\mathfrak{d}} \mathfrak{d}\left(\ln \left\|u_{1}\right\|_{\mathfrak{o}}\right) f\left(z_{1}\right)-$ $\left\|u_{2}\right\|_{\mathfrak{d}} \mathfrak{d}\left(\ln \left\|u_{2}\right\|_{\mathfrak{d}}\right) f\left(z_{2}\right)=\left\|u_{1}\right\|_{\mathfrak{d}}^{\mathfrak{V}} \mathfrak{d}\left(\ln \left\|u_{1}\right\|_{\mathfrak{d}}\right)\left(f\left(z_{1}\right)-f\left(z_{2}\right)\right)+\left(\left\|u_{1}\right\|_{\mathfrak{d}} \mathfrak{d}\left(\ln \left\|u_{1}\right\|_{\mathfrak{o}}\right)-\right.$ $\left\|u_{2}\right\|^{v} \mathfrak{d}\left(\ln \left\|u_{1}\right\|_{\mathfrak{d}}\right) f\left(z_{2}\right)+\left(\left\|u_{2}\right\|^{v} \mathfrak{d}\left(\ln \left\|u_{1}\right\|_{\mathfrak{o}}\right)-\left\|u_{2}\right\|_{\mathfrak{d}}^{v} \mathfrak{d}\left(\ln \left\|u_{2}\right\|_{\mathfrak{o}}\right)\right) f\left(z_{2}\right)$. If $L>0$ is a Lipschitz constant on $S$ then $\left\|f\left(u_{1}\right)-f\left(u_{2}\right)\right\| \leq L\left\|u_{1}\right\|_{\mathfrak{d}}^{\mathfrak{v}} \mathfrak{d}\left(\ln \left\|u_{1}\right\|_{\mathfrak{o}}\right)\left\|z_{1}-z_{2}\right\|+$ $\left\|\mathfrak{d}\left(\ln \left\|u_{1}\right\|_{\mathfrak{o}}\right) f\left(z_{2}\right)\right\|\left(\left\|u_{1}\right\|_{\mathfrak{d}}^{v}-\left\|u_{2}\right\|_{\mathfrak{d}}^{v}\right)+\left\|f\left(z_{2}\right)\right\|\left\|u_{2}\right\|^{v}\left\|\mathfrak{d}\left(\ln \left\|u_{1}\right\|_{\mathfrak{o}}\right)-\mathfrak{d}\left(\ln \left\|u_{2}\right\|_{\mathfrak{d}}\right)\right\|_{\mathbb{A}}$. Since $\mathfrak{d}\left(s_{1}\right)-\mathfrak{d}\left(s_{2}\right)=G_{\mathfrak{d}} \int_{s_{2}}^{s_{1}} \mathfrak{d}(s) d s$ and the function $\|\mathfrak{d}(\cdot)\|_{\mathbb{A}}$ is strictly monotone increasing then $\left.\| \mathfrak{d}\left(\ln \left\|u_{1}\right\|_{\mathfrak{d}}\right)-\mathfrak{d}\left(\ln \left\|u_{2}\right\|_{\mathfrak{d}}\right)\right)\left\|_{\mathbb{A}} \leq M\right\| G_{\mathfrak{d}}\|\mid \ln \| u_{1}\left\|_{\mathfrak{d}}-\ln \right\| u_{2} \|_{\mathfrak{d}}$, where $M=\max \left\{\left\|\mathfrak{d}\left(\ln \left\|u_{1}\right\|_{\mathfrak{d}}\right)\right\|_{\mathbb{A}},\left\|\mathfrak{d}\left(\ln \left\|u_{2}\right\|_{\mathfrak{o}}\right)\right\|_{\mathbb{A}}\right\}$. Since the homogeneous norm is Lipschitz continuous (see Proposition 3) on $\mathbb{R}^{n} \backslash\{\boldsymbol{0}\}$ and power and logarithm functions are Lipschitz continuous outside zero then $f$ is Lipschitz continuous outside the origin.

Necessity. Suppose the contrary, i.e. $f$ is Lipschitz continuous on $\mathbb{R}^{n} \backslash\{\boldsymbol{0}\}$, but it does not satisfy the Lipschitz condition of $S$. This means for any $L_{n}>0$ there exists $u_{n}, v_{n} \in S$ such that $\left\|f\left(u_{n}\right)-f\left(v_{n}\right)\right\|>L_{n}\left\|u_{n}-v_{n}\right\|, n=1,2, .$. , i.e. $L_{n} \rightarrow+\infty$ as $n \rightarrow+\infty$. Since $S$ is compact then $f$ is bounded on $S$. This means that $\left\|u_{n}-v_{n}\right\| \rightarrow 0$ as $n \rightarrow 0$. The latter contradicts to Lipschitz continuity, since for any $u \in S$ there exists $\varepsilon_{u}>0$ and $L_{u}>0$ such that $\|u-v\|<\varepsilon$ implies $\|f(u)-f(v)\| \leq L_{u}\|u-v\|$.

iv) Since $h(u)=h\left(\mathfrak{d}\left(\ln \|u\|_{\mathfrak{o}}\right) z\right)=\|u\|_{\mathfrak{d}}^{v} h(z)$ where $z=\mathfrak{d}(-\ln \|u\|) u \in S$ then $\frac{\partial h}{\partial u}=\frac{\partial}{\partial u}\left(\|u\|_{\mathfrak{d}}^{v} h(z)\right), z=z(u)=\mathfrak{d}\left(-\ln \|u\|_{\mathfrak{o}}\right) u$. Since $\|\cdot\|_{\mathfrak{o}}$ is smooth outside the origin then the differentiability of the function $h$ on the sphere $S$ implies its differentiability on $\mathbb{R}^{n} \backslash\{\boldsymbol{0}\}$. From $h(u)=\|u\|_{\mathfrak{d}}^{v} h\left(\mathfrak{d}\left(-\ln \|u\|_{\mathfrak{d}}\right) u\right)$ we derive $\frac{\partial h(u)}{\partial u}=v h$ $\left(\mathfrak{d}\left(-\ln \|u\|_{\mathfrak{o}}\right) u\right)\|u\|_{\mathfrak{d}}^{v-1} \frac{\partial\|u\|_{\mathfrak{d}}}{\partial u}+\|u\|_{\mathfrak{d}}^{v} \frac{\partial h\left(\mathfrak{d}\left(-\ln \|u\|_{\mathfrak{o}} u\right)\right)}{\partial u}=v h\left(\mathfrak{d}\left(-\ln \|u\|_{\mathfrak{d}}\right) u\right)\|u\|_{\mathfrak{d}}^{v-1} \frac{\partial\|u\|_{\mathfrak{o}}}{\partial u}$ $+\left.\|u\|_{\mathfrak{d}}^{v} \frac{\partial h(z)}{\partial z}\right|_{z=\mathfrak{d}\left(-\ln \|u\|_{\mathfrak{d}}\right) u} \frac{\partial}{\partial u}\left(\mathfrak{d}\left(-\ln \|u\|_{\mathfrak{o}}\right) u\right)=v h\left(\mathfrak{d}\left(-\ln \|u\|_{\mathfrak{d}} u\right)\|u\|_{\mathfrak{d}}^{v-1} \frac{\partial\|u\|_{\mathfrak{d}}}{\partial u}+\right.$ 
$\left.\|u\|_{\mathfrak{d}}^{v} \frac{\partial h(z)}{\partial z}\right|_{z=\mathfrak{d}\left(-\ln \|u\|_{\mathfrak{d}}\right) u}\left(\mathfrak{d}\left(-\ln \|u\|_{\mathfrak{d}}\right)-\frac{G_{\mathfrak{d}} \mathfrak{d}\left(-\ln \|u\|_{\mathfrak{d}}\right) u}{\|u\|_{\mathfrak{d}}} \frac{\partial\|u\|_{\mathfrak{o}}}{\partial u}\right)$. For $\|u\|=1$ we derive $\frac{\partial h(u)}{\partial u} G_{\mathfrak{d}} u \frac{\partial\|u\|_{\mathfrak{o}}}{\partial u}=v h(u) \frac{\partial\|u\|_{\mathfrak{o}}}{\partial u}$. Hence, multiplying by $u$ we derive that (11) holds for $\|u\|=1$. Since $\|\cdot\|_{\text {new }}=\gamma\|\cdot\|$ with $\gamma>0$ is again the norm satisfying Theorem 2 then the obtained identity holds on $\mathbb{R}^{n} \backslash\{0\}$. Finally, note that this identity immediately implies homogeneity of the gradient field of $h$.

Let $\operatorname{deg}_{\mathfrak{d}}(h)$ (resp. $\operatorname{deg}_{\mathfrak{d}}(f)$ ) denote the homogeneity degree of $\mathfrak{d}$ homogeneous function $h$ (resp. $\mathfrak{d}$-homogeneous vector field $f$ ).

Theorem 3 ('Homogeneous arithmetics"). If $h, w$ are $\mathfrak{d}=$ homogeneous functions and $f, g$ are vector fields then

1. if $\operatorname{deg}_{\mathfrak{d}}(h)=\operatorname{deg}(w)$ then $\operatorname{deg}_{\mathfrak{d}}(w+h)=\operatorname{deg}_{\mathfrak{d}}(w)=\operatorname{deg}_{\mathfrak{d}}(h)$;

2. $\operatorname{deg}_{\mathfrak{d}}(w h)=\operatorname{deg}_{\mathfrak{d}}(w)+\operatorname{deg}_{\mathfrak{d}}(h)$;

3. if $h \in C^{1}$ at least on $\mathbb{R}^{n} \backslash\{\boldsymbol{0}\}$ then

$$
e^{\operatorname{deg}(h) s} \frac{\partial h(u)}{\partial u}=\left.\frac{\partial h(z)}{\partial z}\right|_{z=\mathfrak{d}(s) u} \mathfrak{d}(s), \quad \forall u \in \mathbb{R}^{n} \backslash\{\boldsymbol{0}\}, \quad \forall s \in \mathbb{R} ;
$$

4. if $\operatorname{deg}_{\mathfrak{d}}(f)=\operatorname{deg}_{\mathfrak{d}}(g)$ then $\operatorname{deg}_{\mathfrak{d}}(f+g)=\operatorname{deg}_{\mathfrak{d}}(f)=\operatorname{deg}_{\mathfrak{d}}(g)$;

5. $\frac{\partial h}{\partial u} f \in \mathbb{H}_{\mathfrak{d}}$ and $\operatorname{deg}_{\mathfrak{d}}\left(\frac{\partial h}{\partial u} f\right)=\operatorname{deg}_{\mathfrak{d}}(h)+\operatorname{deg}_{\mathfrak{d}}(f)$ if $h \in C^{1}\left(\mathbb{R}^{n} \backslash\{\boldsymbol{0}\}, \mathbb{R}\right)$.

Proof. The properties 1,2, and 4 are obvious. The property 3 follow from the definition of the Frechét derivative, which coincides with $\frac{\partial h}{\partial u}$ if $h$ is smooth. Namely, $\lim _{\|\Delta\| \rightarrow 0} \frac{\left\|h(u+\Delta)-h(u)-\frac{\partial h(u)}{\partial u} \Delta\right\|}{\|\Delta\|}=0$ and $\lim _{\|\Delta\| \rightarrow 0} \frac{\left\|h(\mathfrak{d}(s) u+\Delta)-h(\mathfrak{d}(s) u)-\left.\frac{\partial h(z)}{\partial z}\right|_{z=\mathfrak{d}(s) u} \Delta\right\|}{\|\Delta\|}=$ 0 with $\Delta \in \mathbb{R}^{n}$. Since $h$ is $\mathfrak{d}$-homogeneous then $\frac{\left\|h(\mathfrak{d}(s) u+\Delta)-h(\mathfrak{d}(s) u)-\left.\frac{\partial h(z)}{\partial z}\right|_{z=\mathfrak{d}(s) u} \Delta\right\|}{\|\Delta\|}=$ $e^{v s} \frac{\left\|h(u+\tilde{\Delta})-h(u)-\left.e^{-v s} \frac{\partial h(z)}{\partial z}\right|_{z=\mathfrak{d}(s) u} \mathfrak{d}(s) \tilde{\Delta}\right\|}{\|\mathfrak{d}(s) \tilde{\Delta}\|} \leq \frac{e^{v s}}{\mathfrak{d}(s)\rfloor_{\mathbb{A}}} \frac{\left\|h(u+\tilde{\Delta})-h(u)-\left.e^{-v s} \frac{\partial h(z)}{\partial z}\right|_{z=\mathfrak{d}(s) u} \mathfrak{d}(s) \tilde{\Delta}\right\|}{\|\tilde{\Delta}\|}$, where $\tilde{\Delta}=\mathfrak{d}(-s) \Delta$ such that $\|\tilde{\Delta}\| \rightarrow 0$ implies $\|\Delta\| \rightarrow 0$. Therefore the identity (12) holds. The property 5 is the straightforward corollary of the property 3 .

Let us denote by $\mathscr{L}_{f}^{(k)} h$ the Lie derivative of the function along the vector field $f$, i.e. $\mathscr{L}_{f}^{(0)} h=h$ and $\mathscr{L}_{f}^{(k)} h=\frac{\partial \mathscr{L}_{f}^{(k-1)} h}{\partial u} f$ for $k=1,2, \ldots$.

Corollary 2. If $\mathfrak{d}$-homogeneous vector field $f: \mathbb{R}^{n} \rightarrow \mathbb{R}^{n}$ and $\mathfrak{d}$-homogeneous function $h: \mathbb{R}^{n} \rightarrow \mathbb{R}$ are sufficiently smooth to guarantee existence and continuity the Lie derivatives up to the order $k$ at least on $\mathbb{R}^{n} \backslash\{\boldsymbol{0}\}$ then $\mathscr{L}_{f}^{(i)} h$ is $\mathfrak{d}$-homogeneous of degree $\operatorname{deg}_{\mathfrak{d}}(g) i+\operatorname{deg}_{\mathfrak{d}}(h), i=0,1,2, \ldots$. 


\subsection{Homogeneous Differential Equations}

\subsubsection{Stability of Homogenenous Systems}

The next theorem gives the most important result about scalability solutions to $\mathfrak{d}$ homogeneous evolution equations [49], [23], [41], [7], [28], [35].

Theorem 4. Let $f: \mathbb{R}^{n} \rightarrow \mathbb{R}^{n}$ be continuous $\mathfrak{d}$ - homogeneous vector field of degree $v \in \mathbb{R}$. If $\varphi_{x_{0}}: \mathbb{R}_{+} \rightarrow \mathbb{R}^{n}$ is a solution to

$$
\dot{x}=f(x),
$$

with the initial condition $x(0)=x_{0} \in \mathbb{R}^{n}$ then $\varphi_{\mathfrak{d}(s) x_{0}}(t):=\mathfrak{d}(s) \varphi\left(t_{0}+e^{v s} t\right)$ with $t_{0}, s \in \mathbb{R}$ is a solution to (13) with the initial condition $x(0)=\mathfrak{d}(s) \varphi_{x_{0}}\left(t_{0}\right)$.

Proof. Since $\frac{d}{d t} \varphi(t)=f(\varphi(t))$ then $\mathfrak{d}(s) \frac{d}{d t} \varphi(t)=\frac{d}{d t} \mathfrak{d}(s) \varphi(t)=\mathfrak{d}(s) f(\varphi(t))=$ $e^{-v s} f(\mathfrak{d}(s) \varphi(t))$. Making the change of time $t=t_{0}+e^{v s} t^{n e w}$ we complete the proof.

This theorem has a lot of corollaries, which are very useful for qualitative analysis of homogeneous systems.

Corollary 3. Let a continuous vector field $f: \mathbb{R}^{n} \rightarrow \mathbb{R}^{n}$ be $\mathfrak{d}$ - homogeneous of degree $v \in \mathbb{R}$. The origin of the system (13) is globally uniformly asymptotically stable if and only if one of the following three conditions holds

1) it is locally attractive;

2) there exists a strictly positively invariant compact set ${ }^{2}$ to the system (13);

3) there exists a d-homogeneous Lyapunov function $V: \mathbb{R}^{n} \rightarrow \mathbb{R}_{+}$such that $V \in C^{\infty}$.

Proof. 1) Necessity is obvious. Let us prove Sufficiency. Local attractivity implies that there exists a closed ball $\bar{B}$ with the center at the origin such that $\varphi_{x_{0}}(t) \rightarrow \mathbf{0}$ as $t \rightarrow+\infty$ for any $x_{0} \in B$. Theorem 4 implies that the same property holds for any $\mathfrak{d}$ homogeneous ball $\mathfrak{d}(s) \bar{B}$, i.e. the origin is globally attractive. All trajectories of the system with initial conditions $x_{0} \in \bar{B}$ and $t \in\left[0, t^{*}\right]$ form again a compact set $\bar{\Omega}_{t^{*}}$. Since the origin is globally attractive then the set $\bar{\Omega}=\bigcup_{t_{*} \geq 0} \bar{\Omega}_{t^{*}}$ is compact invariant set to the system (13). Using Theorem 4 we derive the same result for $\mathfrak{d}(s) \bar{\Omega}$, i.e. the origin of the system (13) is Lyapunov stable and, consequently, globally uniformly asymptotically stable.

2) Necessity follows from the Converse Lyapunov theorem (see [?]) about existence of the Lyapunov function for any uniformly asymptotically stable continuous system. The level set of the corresponding Lyapunov function is strictly positively invariant compact set. Sufficiency. Since $f$ is continuous and $\mathfrak{d}$-homogeneous then $f(\mathbf{0})=\mathbf{0}$, i.e. the origin is the equilibrium point of the system (13). If $\Omega$ is strictly positively invariant then by Theorem 4 the set $\mathfrak{d}(s) \Omega$ as well as is also strictly positively invariant compact set for the system (13). This means that

${ }^{2}$ A compact set $\Omega$ is strictly positively invariant for (13) if $x_{0} \in \partial \Omega \Rightarrow \varphi_{x_{0}}(t) \in \operatorname{int}(\Omega), t>0$. 
$0 \in \operatorname{int} \Omega$. Indeed, otherwise there exists $s^{*}$ such that $\operatorname{int}(\mathfrak{d}(s) \Omega) \cap \operatorname{int}(\Omega)=\emptyset$ and $\partial(\mathfrak{d}(s) \Omega) \cap \partial(\Omega) \neq \emptyset$, but the latter contradicts strict positive invariance of these sets. On the other hand, the origin is the unique equilibrium of the system (13). Indeed, otherwise, $f\left(\mathfrak{d}(s) x^{*}\right)=0$ for all $s \in \mathbb{R}$ if $f\left(x^{*}\right)=0$ and the continuous curve $\left\{\mathfrak{d}(s) x^{*}: s \in \mathbb{R}\right\}$ crosses $\Omega$, but this again contradicts strict positive invariance of $\Omega$. Taking into account continuity of the dilation $\mathfrak{d}$ and $\mathfrak{d}(s) \Omega \rightarrow \mathbf{0}$ as $s \rightarrow 0$ we conclude that the origin is locally attractive and globally asymptotically stable.

3) Sufficiency is obvious. Let us prove necessity using the idea proposed in [41]. The Converse Lyapunov Theorem implies that there exists a smooth Lyapunov function $V: \mathbb{R}^{n} \rightarrow \mathbb{R}_{+}$. Let the smooth function $a: \mathbb{R} \rightarrow \mathbb{R}_{+}$be defined as $a(\rho)=e^{\frac{1}{1-\rho}}$ if $\rho>0$ and $a(\rho)=0$ if $\rho \leq 1$. Obviously, $a^{\prime}(\rho)>0$ if $\rho>1$. Then the function $V_{\text {hom }}: \mathbb{R}^{n} \rightarrow \mathbb{R}_{+}$defined as $V_{\text {hom }}(x)=\int_{-\infty}^{+\infty} e^{-s} a(V(\mathfrak{d}(s) x)) d s$ is $\mathfrak{d}$-homogeneous Lyapunov function to the system (13). Indeed,it is well-defined due to cut-off function $a$ ), smooth, positive definite and radially unbounded. Finally, $V$ is $\mathfrak{d}$-homogeneous $V(\mathfrak{d}(q) x)=\int_{-\infty}^{+\infty} e^{-s} a(V(\mathfrak{d}(-s+q) x)) d s=e^{q} V(x)$ and $\dot{V}(x)=$ $\left.\int_{-\infty}^{+\infty} e^{-s} a^{\prime}(V(\mathfrak{d}(-s) x)) \frac{\partial V(z)}{\partial z}\right|_{z=\mathfrak{d}(s) x} \mathfrak{d}(s) f(x) d s<0$ since $f$ is $\mathfrak{d}$-homogeneous and $\left.\frac{\partial V(z)}{\partial z}\right|_{z=\mathfrak{d}(s) x} \mathfrak{d}(s) f(x)=\left.e^{-v s} \frac{\partial V(z)}{\partial z} f(z)\right|_{z=\mathfrak{d}(s) x}<0$.

Corollary 4. Let a continuous vector field $f: \mathbb{R}^{n} \rightarrow \mathbb{R}^{n}$ be $\mathfrak{d}$ - homogeneous of degree $v \in \mathbb{R}$ and the origin of (13) be globally uniformly asymptotically stable.

- If $v<0$ then it is globally finite-time stable.

- If $v>0$ then any ball $B_{\mathfrak{d}}(r)$ is fixed-time attractive, i.e.

$$
\forall r>0 \quad \exists T=T(r) \geq 0 \quad: \quad \varphi_{x_{0}}(t) \in B_{\mathfrak{d}}(r), \quad \forall t>T, \quad \forall x_{0} \in \mathbb{R}^{n} .
$$

Proof. Let us denote $T_{0}=\sup _{\left\|x_{0}\right\|_{\mathfrak{d}} \leq 2} \inf _{\tau \geq 0:\left\|\varphi_{x_{0}}(t)\right\|_{\mathfrak{d}} \leq 1, \forall t>\tau} \tau$. Since the oringin is uniformly asymptotically stable then $T_{0}<+\infty$ and $\varphi_{x_{1}}(t):=\mathfrak{d}(\ln 2) \varphi_{x_{0}}\left(T_{0}+2^{v} t\right)$ is a solution to (13) with $x(0)=x_{1}:=\mathfrak{d}(\ln 2) \varphi_{x_{0}}\left(T_{0}\right)$ such that $\left\|\varphi_{x_{1}}(t)\right\|_{\mathfrak{o}} \leq 2$ for all $t \geq$ 0 . Hence, $\varphi_{x_{2}}(t):=\mathfrak{d}(\ln 2) \varphi_{x_{1}}\left(T_{0}+2^{v} t\right)=\mathfrak{d}(2 \ln 2) \varphi_{x_{0}}\left(T_{0}+2^{v}\left(T_{0}+2^{v} t\right)\right)$ is again a solution to (13) $x(0)=x_{2}:=\mathfrak{d}(\ln 2) \varphi_{x_{1}}\left(T_{0}\right)$ such that $\left\|\varphi_{x_{2}}(t)\right\|_{\mathfrak{d}} \leq 2$. We derive $\varphi_{x_{i}}(t)=\mathfrak{d}(i \ln 2) \varphi_{x_{0}}\left(2^{i v} t+T_{0} \sum_{j=0}^{i-1} 2^{j v}\right),\left\|\varphi_{x_{i}}\left(T_{0}\right)\right\|_{\mathfrak{d}}=\left\|\mathfrak{d}(i \ln 2) \varphi_{x_{0}}\left(T_{0} \sum_{j=0}^{i} 2^{j v}\right)\right\|_{\mathfrak{d}}$ $=2^{i}\left\|\varphi_{x_{0}}\left(T_{i}\right)\right\|_{\mathfrak{d}} \leq 1$, where $T_{i}=T_{0} \sum_{j=0}^{i} 2^{j v}$. Hence, $\left\|\varphi_{x_{0}}\left(T_{i}\right)\right\|_{\mathfrak{o}} \rightarrow 0$ as $i \rightarrow+\infty$, but $T_{i} \rightarrow T:=T_{0} \sum_{j=0}^{+\infty} 2^{j v}$ as $i \rightarrow+\infty$. For $v<0$ we have $T=\frac{T_{0}}{1-2^{v}}<+\infty$.

Since $\left.\varphi_{\tilde{x}_{1}}(t)=\mathfrak{d}(\ln 2)\right) \varphi_{x_{0}}\left(2^{v} t\right)$ is a solution to (13) with $x(0)=\tilde{x}_{1}:=\mathfrak{d}(\ln 2) x_{0}$ then we have $\left\|\varphi_{\tilde{x}_{1}}(t)\right\|_{\mathfrak{d}}=2\left\|\varphi_{x_{0}}\left(2^{v} t\right)\right\|_{\mathfrak{o}} \leq 2$ for $t \geq 2^{-v} T_{0}$ and $\left\|\varphi_{\tilde{x}_{1}}(t)\right\|_{\mathfrak{d}} \leq 1$ for $t \geq 2^{-v} T_{0}+T_{0}$ due to definition of $T_{0}$. Similarly, $\left.\varphi_{\tilde{x}_{i}}(t)=\mathfrak{d}(\ln 2)\right) \varphi_{\tilde{x}_{i-1}}\left(2^{v} t\right)$ is a solution to (13) with $x(0)=\tilde{x}_{2}:=\mathfrak{d}(\ln 2) \tilde{x}_{1}$ and $\left\|\varphi_{\tilde{x}_{i}}(t)\right\|_{\mathfrak{o}} \leq 1$ for all $t \geq T_{0} \sum_{j=0}^{i} 2^{-j v}$ as $i \rightarrow+\infty$. We complete the proof with the remark: $\left\|\tilde{x}_{i}\right\|_{\mathfrak{o}} \rightarrow+\infty$ as $i \rightarrow+\infty$ but $T^{*}=T_{0} \sum_{j=0}^{+\infty} 2^{-j v}<+\infty$ if $v>0$. 


\subsubsection{Observability of Homogeneous Systems}

Let us consider the nonlinear system

$$
\dot{x}=f(x), \quad x \in \mathbb{R}^{n}, \quad y=h(x), \quad y \in \mathbb{R},
$$

The points $x_{1}, x_{2} \in \mathbb{R}^{n}, x_{1} \neq x_{2}$ are indistinguishable if $h\left(\varphi_{x_{1}}(t)\right)=h\left(\varphi_{x_{2}}(t)\right)$ for all $t \geq 0$. Otherwise, these points are said to be distinguishable.

Definition 15 (Observability). The system (14) is locally observable at the point $x_{0} \in \mathbb{R}^{n}$ if there exists a neighborhood $U\left(x_{0}\right)$ such that for any $y \in U\left(x_{0}\right) \backslash\left\{x_{0}\right\}$ the points $x_{0}$ and $y$ are distinguishable. The system (14) is globally observable in $\mathbb{R}^{n}$ if it is locally observable at any $x_{0} \in \mathbb{R}^{n}$.

Corollary 5. Let $f: \mathbb{R}^{n} \rightarrow \mathbb{R}^{n}$ be $\mathfrak{d}$ - homogeneous continuous vector field and $h: \mathbb{R}^{n} \rightarrow \mathbb{R}$ be d-homogeneous continuous function. The nonlinear system (14) is observable on $\mathbb{R}^{n} \backslash\{\boldsymbol{0}\}$ if and only if it is locally observable on the sphere $S$.

This corollary is the straightforward consequence of Definition 15 and Theorem 4.

Theorem 5. Let the norm $\|\cdot\|$ be smooth in $\mathbb{R}^{n} \backslash\{\boldsymbol{0}\}$ and the dilation $\mathfrak{d}$ be strictly monotone on $\mathbb{R}^{n}$. Let $h: \mathbb{R}^{n} \rightarrow \mathbb{R}$ be d-homogeneous of degree $\mu>0$ and $f: \mathbb{R}^{n} \rightarrow \mathbb{R}^{n}$ be $\mathfrak{d}$ - homogeneous of degree $v>-\frac{\mu}{n-1}$. Let the vector field $H=\left(\begin{array}{c}h \\ L_{f} h \\ \cdots \\ L_{f}^{(n-1)} h\end{array}\right)$ : $\mathbb{R}^{n} \rightarrow \mathbb{R}^{n}$ be $C^{1}$ on $S$. The nonlinear system (14) is globally observable if one of the following conditions holds

i) $n \geq 3, \quad \inf _{u \in S}\|H(u)\|>0$ and $\operatorname{det}\left(\left.\frac{\partial H}{\partial u}\right|_{u \in S}\right) \neq 0$;

ii) $H$ is $C^{1}$ at zero, $\operatorname{det}\left(\left.\frac{\partial H}{\partial u}\right|_{u=0}\right) \neq 0$ and $n \mu+\frac{n(n-1)}{2} v=\operatorname{trace}\left(G_{\mathfrak{o}}\right)$.

Proof. To guarantee observability it is sufficient to show that the map $H$ is homeomorphism (or diffeomorphism) on $\mathbb{R}^{n}$.

We have that $L_{f}^{(i)} h$ is homogeneous of degree $\mu+i v$ (see, Corollary 2). Hence,

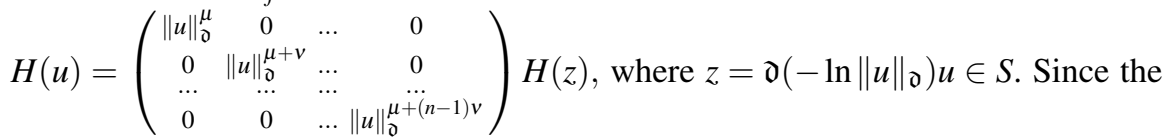
norm $\|\cdot\|$ is selected to be smooth on $\mathbb{R}^{n}$ and $H$ is $C^{1}$ on the sphere $S$ then $H$ is $C^{1}$ on $\mathbb{R}^{n} \backslash\{\boldsymbol{0}\}$ (see, Corollary 1). Due to (12) we derive $\left(\begin{array}{cccc}e^{\mu s} & 0 & \ldots & 0 \\ 0 & e^{(\mu+v) s} & \ldots & 0 \\ \ldots & \ldots & \ldots & \ldots \\ 0 & 0 & \ldots & e^{(\mu+(n-1) v) s}\end{array}\right) \frac{\partial H(u)}{\partial u}=$ $\frac{\partial H(\mathfrak{d}(s) u)}{\partial u} \mathfrak{d}(s)$ and $\frac{e^{(n \mu+0.5 n(n-1) v) s} \operatorname{det}\left(\frac{\partial H(u)}{\partial u}\right)}{\operatorname{det}(\mathfrak{d}(s))}=\operatorname{det}\left(\frac{\partial H(\mathfrak{d}(s) u)}{\partial u}\right)$. Note that $\operatorname{det}(\mathfrak{d}(s))=$ $\operatorname{det}\left(e^{G_{\mathfrak{d}} s}\right)=e^{s \operatorname{trace}\left(G_{\mathfrak{d}}\right)}$ for all $s \in \mathbb{R}$.

i) Therefore, $\left.\operatorname{det}\left(\frac{\partial H(u)}{\partial u}\right) \neq 0\right)$ on $\mathbb{R}^{n} \backslash\{\mathbf{0}\}$. On the other hand, since $H(z) \neq \mathbf{0}$ for $z \in S$ then $\|H(u)\| \rightarrow+\infty$ as $\|u\| \rightarrow+\infty$ (i.e. $H$ radially unbounded and, consequently, proper) and $\|H(u)\| \rightarrow 0$ as $\|u\| \rightarrow 0$ (i.e. $H$ is continuous at zero). According Theorem of Hadamard (see, e.g. Theorem 2.1, [45]) we derive that $H$ is the 
bijection on $\mathbb{R}^{n} \backslash\{\boldsymbol{0}\}$ provided that $\mathbb{R}^{n} \backslash\{\boldsymbol{0}\}$ is simply connected $C^{1}$-manifold (that is the case for $n \geq 3$ ). Continuity of $H$ at the origin proven above as well as $H(\mathbf{0})=\mathbf{0}$ together with $H(u) \neq \mathbf{0}$ on $\mathbb{R}^{n} \backslash\{\mathbf{0}\}$ implies that $H$ is the global homeomorphism.

ii) If $n \mu+\frac{n(n-1)}{2} v=\operatorname{trace}\left(G_{\mathfrak{D}}\right)$ then $\operatorname{det}\left(\frac{\partial H(u)}{\partial u}\right)=\operatorname{det}\left(\frac{\partial H(\mathfrak{o}(s) u)}{\partial u}\right)$ for all $s \in \mathbb{R}$.

Since $H$ is $C^{1}$ at zero then for all $u \in \mathbb{R}^{n}$ we have $\operatorname{det}\left(\frac{\partial H(u)}{\partial u}\right)=\operatorname{det}\left(\left.\frac{\partial H}{\partial u}\right|_{u=0}\right) \neq 0$, i.e. $H$ is $C^{1}$ diffeomorphism on $\mathbb{R}^{n}$.

The condition ii) of Theorem 5 covers linear systems if $v=0, \mu=1$ and $G_{\mathfrak{d}}=I$.

\subsection{Implicit Lyapunov Function Method}

Lyapunov function method [27] is the main tool for analysis of nonlinear dynamical systems. Frequently, it is very difficult to find an appropriate Lyapunov function. However, stability analysis of homogeneous differential equation can be reduced to convergence analysis from a sphere $S$. Indeed, this sphere can be assigned to be the unit level set of a Lyapunov function, which can be constructed by means of the homogeneous dilation of $S$ yielding $\mathfrak{d}(s) S$ to be its $e^{s}$-level set. The latter implies that the Lyapunov function of homogeneous system can always be designed implicitly

$$
V: \mathbb{R}^{n} \rightarrow \mathbb{R}_{+} \quad \text { such that } \quad \mathfrak{d}(-\ln V(x)) x \in S, \quad x \in \mathbb{R} \backslash\{\boldsymbol{0}\} .
$$

using on the so-called Implicit Lyapunov Function method [25], [1], [36].

Theorem 6. [36] Let continuous function $Q: \mathbb{R}_{+} \times \mathbb{R}^{n} \rightarrow \mathbb{R}$ satisfy the conditions

C1) $Q$ is continuously differentiable outside the origin of $\mathbb{R}_{+} \times \mathbb{R}^{n}$;

C2) for any $x \in \mathbb{R}^{n} \backslash\{0\}$ there exists $V \in \mathbb{R}_{+}$such that $Q(V, x)=0$;

C3) $\lim _{\substack{x \rightarrow 0 \\(V, x) \in \Omega}} V=0, \lim _{\substack{V \rightarrow 0^{+} \\(V, x) \in \Omega}}\|x\|=0, \lim _{\substack{\|x\| \rightarrow \infty \\(V, x) \in \Omega}} V=+\infty$, where $\Omega=\left\{(V, x) \in \mathbb{R}_{+} \times \mathbb{R}^{n}: Q(V, x)=0\right\}$;

C4) $\frac{\partial Q(V, x)}{\partial V}<0$ for all $V \in \mathbb{R}_{+}$and $x \in \mathbb{R}^{n} \backslash\{0\}$.

If $\frac{\partial Q(V, x)}{\partial x} f(x)<0$ for all $(V, x) \in \Omega$ then the origin of (13) is globally uniformly asymptotically stable.

Proof. The conditions $(1), C 2$ ), C4) and the implicit function theorem [12] imply that the equation $Q(V, x)=0$ implicitly defines a unique positive definite function $V: \mathbb{R}^{n} \backslash\{0\} \rightarrow \mathbb{R}_{+}$such that $Q(V(x), x)=0$ for all $x \in \mathbb{R}^{n} \backslash\{0\}$. The function $V$ is continuously differentiable outside the origin and $\frac{\partial V}{\partial x}=-\left[\frac{\partial Q(V, x)}{\partial V}\right]^{-1} \frac{\partial Q(V, x)}{\partial x}$ for $Q(V, x)=0, x \neq 0$. Hence, since $\frac{\partial Q(V, x)}{\partial V}<0$ then the condition $\frac{\partial Q(V, x)}{\partial x} f(x)<0$ implies $\dot{V}(x)=\frac{\partial V}{\partial x} f(x)<0$. Note that due to the condition $\left.C 3\right)$ the function $V$ is radially unbounded and it can be continuously prolonged at the origin by $V(0)=0$.

Evidently, the conditions of Theorem 6 mainly repeat (in the implicit form) the requirements of the classical theorem on global asymptotic stability (see, for example, [4]). Indeed, Condition C1) asks for smoothness of the Lyapunov function. 
Condition $\mathrm{C2}$ ) and the first two limits from Condition $\mathrm{C3}$ ) provide its positive definiteness. The last limit from Condition C3) implies radial unboundedness of the Lyapunov function. Condition $C 5$ ) guarantees the negative definiteness of the total derivative of the Lyapunov function calculated along trajectories of the system (13). The only specific condition is $C 4$ ), which is imposed by implicit function theorem (see, for example, [12]). This condition is required in order to guarantee that the Lyapunov function is (uniquely) well-defined by the equation $Q(V, x)=0$.

Corollary 6. [36] Let a continuous function $Q: \mathbb{R}_{+} \times \mathbb{R}^{n} \rightarrow \mathbb{R}$ satisfy the conditions C1)-C4) of Theorem 6. If there exist $c>0$ and $0<\mu \leq 1$ such that $\frac{\partial Q(V, x)}{\partial x} f \leq$ $c V^{1-\mu} \frac{\partial Q(V, x)}{\partial V}$ for $(V, x) \in \Omega$ then the origin of the system (13) is globally uniformly

finite-time stable and $T\left(x_{0}\right) \leq \frac{V_{0}^{\mu}}{c \mu}$, where $Q\left(V_{0}, x_{0}\right)=0$.

Proof. Theorem 6 implies global uniform asymptotic stability of the origin of (13). The uniform finite-time stability of the origin follows from the differential inequality $\dot{V}(x)) \leq-c V^{1-\mu}(x)$, which, due to the condition of this corollary, holds.

Corollary 7. [36] Let there exist two functions $Q_{1}$ and $Q_{2}$ satisfying the conditions C1)-C4) of Theorem 6 and

C5) $Q_{1}(1, x)=Q_{2}(1, x)$ for all $x \in \mathbb{R}^{n} \backslash\{0\}$;

C6) there exist $c_{1}>0$ and $0<\mu<1$ such that the inequality $\frac{\partial Q_{1}}{\partial x} f(x) \leq c_{1} V^{1-\mu} \frac{\partial Q_{1}}{\partial V}$, holds for all $V \in(0,1]$ and $x \in \mathbb{R}^{n} \backslash\{0\}$ satisfying the equation $Q_{1}(V, x)=0$;

C7) there exist $c_{2}>0$ and $v>0$ such that the inequality $\frac{\partial Q_{2}}{\partial x} f(x) \leq c_{2} V^{1+v} \frac{\partial Q_{2}}{\partial V}$, holds for all $V \geq 1$ and $x \in \mathbb{R}^{n} \backslash\{0\}$ satisfying the equation $Q_{2}(V, x)=0$, then the system (13) is globally fixed-time stable with the settling-time estimate $T\left(x_{0}\right) \leq \frac{1}{c_{1} \mu}+\frac{1}{c_{2} v}$.

Proof. Let the two functions $V_{1}$ and $V_{2}$ be defined by the equations $Q_{1}(V, x)=0$ and $Q_{2}(V, x)=0$ (see, the proof of Theorem 6). Consider the sets $\Sigma_{1}=\left\{x \in \mathbb{R}^{n}: V_{1}(x)>1\right\}$, $\Sigma_{2}=\left\{x \in \mathbb{R}^{n}: V_{2}(x)>1\right\}$ and prove that $\Sigma_{1}=\Sigma_{2}$. Suppose the contrary, i.e. $\exists z \in \mathbb{R}^{n}$ such that $z \in \Sigma_{1}$ and $z \notin \Sigma_{2}$. On the one hand, $Q_{1}\left(V_{1}, z\right)=0$ implies $V_{1}>1$ and $Q_{1}(1, z)>Q_{1}\left(V_{1}, z\right)=0$ due to Condition C4). On the other hand, $Q_{2}\left(V_{2}, z\right)=0$ implies $V_{2} \leq 1$ and $Q_{2}(1, z) \leq Q_{2}\left(V_{2}, z\right)=0$. The contradiction follows from C5).

Therefore, due to $C 5$ ) and $C 4$ ) the function $V: \mathbb{R}^{n} \rightarrow \mathbb{R}$ defined by the equality

$$
V(x)=\left\{\begin{array}{clc}
V_{1}(x) & \text { for } & V_{1}(x)<1 \\
V_{2}(x) & \text { for } & V_{2}(x)>1 \\
1 & \text { for } & V_{1}(x)=V_{2}(x)=1
\end{array}\right.
$$

is positive definite, continuous in $\mathbb{R}^{n}$ and continuously differentiable for $x \notin\{0\} \cup$ $\left\{x \in \mathbb{R}^{n}: V(x)=1\right\}$. The function $V$ is Lipschitz continuous outside the origin and has the following Clarke's gradient [8]:

$$
\nabla_{C} V(x)=\xi \nabla V_{1}(x)+(1-\xi) \nabla V_{2}(x), \quad x \in \mathbb{R}^{n}
$$

where $\xi=1$ for $0<V_{1}(x)<1, \xi=0$ for $V_{2}(x)>1, \xi=[0,1]$ for $V_{1}(x)=V_{2}(x)=1$ and $\nabla V_{i}$ is the gradient of the function $V_{i}, i=1,2$. Hence, due to conditions $\left.C 6\right)$ and $C 7$ ), the inequality

$$
\frac{d V\left(\varphi_{x_{0}}(t)\right)}{d t} \leq\left\{\begin{array}{cl}
-c_{1} V^{1-\mu}\left(\varphi_{x_{0}}(t)\right) & \text { for } V\left(\varphi_{x_{0}}(t)\right)<1 \\
-c_{2} V^{1+v}\left(\varphi_{x_{0}}(t)\right) & \text { for } V\left(\varphi_{x_{0}}(t)\right)>1 \\
-\min \left\{c_{1}, c_{2}\right\} & \text { for } V\left(\varphi_{x_{0}}(t)\right)=1
\end{array}\right.
$$


holds for almost all $t$ such that $\varphi_{x_{0}}(t) \neq 0$, where $\varphi_{x_{0}}(t)$ is a solution of the system (13) with the initial condition $x(0)=x_{0}$. This implies the fixed-time stability of the origin of the system (13) with the estimate of settling-time function given above. Please see [33] or [39] for more details.

Corollary 8. Let $Q_{1}, Q_{2}$ satisfy Conditions C1-C5) of Corollary 7 and $\left.C 6^{*}\right)$ for $\left(V, x_{h}(t)\right) \in \Omega$ such that $x(t)$ satisfies (16) one has

$$
\frac{\partial Q_{i}}{\partial x} \leq 2 \alpha_{0} V \ln \left(e V^{(-1)^{i}}\right) \frac{\partial Q_{i}\left(V, x_{h}(t)\right)}{\partial V}, \quad \forall t \in \mathbb{R}_{+},
$$

where $\alpha_{0}>0$ and $i=1,2$;

then the origin of system (16) is globally hyper exponentially stable with degree $r=1$ and convergence rate $\alpha=\left(\alpha_{0}, 1\right)$.

The proof this corollary is similar to Corollary 7.

The next theorem provides the important topological characterization of homogeneous systems. In particular, it says that the asymptotically stable homogeneous system is isomorphic to some "quadratically stable" system.

Theorem 7. Let $\mathfrak{d}$ be dilation on $\mathbb{R}^{n}$ and $f: \mathbb{R}^{n} \rightarrow \mathbb{R}^{n}$ be a continuous $\mathfrak{d}$-homogeneous vector field. The origin of the system (13) is globally asymptotically stable if and only if there exist a positive definite symmetric matrix $P \in \mathbb{R}^{n \times n}$ satisfying (6) and a d -homogeneous diffeomorphism $\Psi: \mathbb{R}^{n} \backslash\{\boldsymbol{0}\} \rightarrow \mathbb{R}^{n} \backslash\{\boldsymbol{0}\}$ of degree zero such that

$$
\frac{\partial \Psi^{\top}(x) P \Psi(x)}{\partial x} f(x)<0 \quad \text { for } \quad x \in \mathbb{R}^{n}: \Psi^{\top}(x) P \Psi(x)=1 .
$$

Moreover, $\|\Psi(\cdot)\|_{\mathfrak{D}}: \mathbb{R}^{n} \rightarrow \mathbb{R}_{+}$is the $\mathfrak{d}$-homogeneous Lyapunov function of degree 1 , where $\|\cdot\|_{\mathfrak{d}}$ is the $\mathfrak{d}$-homogeneous norm induced by the norm $\|u\|=\sqrt{u^{\top} P u}$.

Proof. Sufficiency. Since $P$ satisfies (6) then the dilation $\mathfrak{d}$ is strictly monotone on $\mathbb{R}^{n}$ equipped with the norm $\|x\|=\sqrt{x^{T} P x}$. Since $\Psi(\mathfrak{d}(s) u)=\mathfrak{d}(s) \Psi(u)$ then $\Psi$ can be prolonged to the origin by continuity $\Psi(\mathbf{0})=\mathbf{0}$. Note also that $\Psi(u) \neq \mathbf{0}$ for all $u \neq 0$, otherwise (i.e. $\exists u^{*} \neq 0: \Psi\left(u^{*}\right)$ ), due to homogeneity we derive that $\Psi(u)=\mathbf{0}$ on a smooth curve $\left\{\mathfrak{d}(s) u^{*}, s \in \mathbb{R}\right\}$, which starts at the origin goes to $\infty$. The latter contradicts the assumption that $\Psi$ is diffeomorphism (continuously differentiable invertible map with continuously differentiable inverse) on $\mathbb{R}^{n} \backslash\{\boldsymbol{0}\}$. Since $\|\Psi(\mathfrak{d}(s) u)\|_{\mathfrak{d}}=\|\mathfrak{d}(s) \Psi(u)\|_{\mathfrak{d}}=e^{s}\|\Psi(u)\|_{\mathfrak{d}}$ then the function $\|\Psi(\cdot)\|_{\mathfrak{d}}$ is $\mathfrak{d}$-homogeneous of degree 1 , radially unbounded, continuous at the origin and continuously differentiable outside the origin. Due to (9) the inequality $\left.\frac{\partial\|\Psi(x)\|}{\partial x} f(x)\right|_{\|\Psi(x)\|=1}<0$ implies $\left.\frac{\partial\|\Psi(x)\|_{\mathrm{o}}}{\partial x} f(x)\right|_{\|\Psi(x)\|=1}<0$. Applying homogeneity we derive $\frac{\partial\|\Psi(x)\|_{\mathfrak{o}}}{\partial x} f(x)<0$ for $x \in \mathbb{R}^{n} \backslash\{\boldsymbol{0}\}$.

Necessity. Since $\mathfrak{d}$ is a dilation on $\mathbb{R}^{n}$ then due to Theorem 2 it is strictly monotone on $\mathbb{R}^{n}$ equipped with the smooth norm $\|x\|=\sqrt{x^{\top} P x}$, where $P>0$ satisfies the inequality (6). Since the origin of the system (13) is asymptotically stable then according to Corollary 3 there exists a smooth $\mathfrak{d}$-homogeneous Lyapunov function 
$\tilde{V}: \mathbb{R}^{n} \rightarrow \mathbb{R}_{+}$of degree $\mu>0$. The function $V=\tilde{V}^{1 / \mu}$ is also Lyapunov function to (13) that is homogeneous of degree 1 , continuous at the origin and smooth outside the origin. Let us consider the map $\Psi: \mathbb{R}^{n} \backslash\{\boldsymbol{0}\} \rightarrow \mathbb{R}^{n} \backslash\{\boldsymbol{0}\}$ defined as $\Psi(x)=$ $\mathfrak{d}\left(\ln \left(\frac{V(x)}{\|x\|_{\mathfrak{o}}}\right)\right) x$ for $x \in \mathbb{R}^{n} \backslash\{\boldsymbol{0}\}$. Obviously, the inverse map $\Psi^{-1}: \mathbb{R}^{n} \backslash\{\boldsymbol{0}\} \rightarrow$ $\mathbb{R}^{n} \backslash\{\boldsymbol{0}\}$ defined as $\Psi^{-1}(z)=\mathfrak{d}\left(-\ln \left(\frac{V(z)}{\|z\|_{\mathfrak{o}}}\right)\right) z$. Finally, $\Psi(\mathfrak{d}(s) x)=\mathfrak{d}(s) \Psi(x)$ and $\|\Psi(x)\|_{\mathfrak{o}}=V(x)$, Using (9) we complete the proof.

Corollary 9. Let $\mathfrak{d}$ be a strictly monotone dilation on $\mathbb{R}^{n}$ equipped with a smooth norm $\|\cdot\|$. Let a map $H: \mathbb{R}^{n} \backslash\{\boldsymbol{0}\} \rightarrow \mathbb{R}^{n} \backslash\{\boldsymbol{0}\}$ be a diffeomorphism and $H(\mathfrak{d}(s) u)=$ $\mathfrak{d}(s) H(u), s \in \mathbb{R}, u \in \mathbb{R}^{n}$. If $f: \mathbb{R}^{n} \rightarrow \mathbb{R}^{n}$ is $\mathfrak{d}$-homogeneous vector field and

$$
\left.\frac{\partial\|H(x)\|}{\partial x} f(x)\right|_{\|H(x)\|=1}<0
$$

then $\|H(\cdot)\|_{\mathfrak{o}}: \mathbb{R}^{n} \rightarrow \mathbb{R}_{+}$is the homogeneous Lyapunov function to the system (13).

Proof. The homogeneous norm is defined implicitly by (8). It satisfies the conditions $\mathrm{C} 1$ )-C3). The condition C4) is also satisfied due to strict monotonicity of $\mathfrak{d}$. Since $H$ is the diffeomorphism on $\mathbb{R}^{n} \backslash\{\boldsymbol{0}\}$ then $\|H \cdot\|_{\mathfrak{d}}$ also satisfy conditions C1)C4). Due to (9) the inequality $\left.\frac{\partial\|H(x)\|}{\partial x} f(x)\right|_{\|H(x)\|=1}<0$ implies $\left.\frac{\partial\|H(x)\|_{\mathrm{o}}}{\partial x} f(x)\right|_{\|x\|=1}<$ 0 . Finally applying homogeneity we derive $\frac{\partial\|H(x)\|_{\mathfrak{o}}}{\partial x} f(x)<0$ for all $x \in \mathbb{R}^{n} \backslash\{\mathbf{0}\}$.

\section{Fast Control for Nonlinear Plants}

Let us consider the nonlinear control system

$$
\begin{gathered}
\dot{x}=f(x, u), \\
y=h(x),
\end{gathered}
$$

where $x \in \mathbb{R}^{n}$ is the state vector of a plant, $u \in \mathbb{R}^{m}$ is the vector of control inputs, $f: \mathbb{R}^{n} \times \mathbb{R}^{m} \rightarrow \mathbb{R}^{n}$ is a continuous vector field of the plant, $y \in \mathbb{R}^{k}$ is a measured output given by means of measurement operator $h: \mathbb{R}^{n} \rightarrow \mathbb{R}^{k}$.

The control aim is to stabilize the origin of the system (16) in a fixed time independently of the initial condition $x(0)=x_{0}$.

Theorem 8 (Dynamical State-Feedback). Let $\overline{\mathfrak{d}}_{1}$ and $\overline{\mathfrak{d}}_{2}$ be dilations in $\mathbb{R}^{n}$ and $\mathfrak{d}_{1}^{*}$ and $\mathfrak{d}_{2}^{*}$ be dilations in $\mathbb{R}^{m}$. Let the joint dilations $\mathfrak{d}_{1}=\left(\begin{array}{l}\overline{\mathfrak{d}}_{1}(s) \\ \mathfrak{d}_{1}^{*}(s)\end{array}\right)$ and $\mathfrak{d}_{2}=\left(\begin{array}{l}\overline{\mathfrak{d}}_{2}(s) \\ \mathfrak{d}_{2}^{*}(s)\end{array}\right)$ be strictly monotone on $\mathbb{R}^{n+m}$ equipped with a smooth norm $\|\cdot\|$. Let the vector field $\tilde{f}=\left(\begin{array}{c}f \\ 0_{m}\end{array}\right): \mathbb{R}^{n+m} \rightarrow \mathbb{R}^{n+m}$ be continuous, $\mathfrak{d}_{1}$-homogeneous of negative degree $\mu_{1}<0$ and $\mathfrak{d}_{2}$-homogeneous of positive degree $\mu_{2}>0$. If there exist $\gamma>0$ and $c>0$ :

$$
\frac{\partial\|\xi\|}{\partial x} f(\xi)+c \leq \gamma \frac{\partial\|\xi\|}{\partial u}\left[\frac{\partial\|\xi\|}{\partial u}\right]^{\top} \text { for }\|\xi\|=1, \quad \xi=\left(\begin{array}{l}
x \\
u
\end{array}\right) \in \mathbb{R}^{n+m}
$$


then the dynamical state feedback

$$
\begin{gathered}
\dot{u}= \begin{cases}g_{1}(\xi) & \text { if }\|\xi\| \leq 1, \quad u(0)=0, \quad \xi=\left(\begin{array}{c}
x \\
u
\end{array}\right) \\
g_{2}(\xi) \text { if }\|\xi\|>1,\end{cases} \\
g_{i}(\xi)=-\gamma\|\xi\|_{\mathfrak{d}_{i}}^{\mu_{i}} \mathfrak{d}_{i}^{*}\left(\ln \|\xi\|_{\mathfrak{d}_{i}}\right)\left[\left.\frac{\partial\|\xi\|}{\partial u}\right|_{\xi=\xi_{i}}\right]^{\top}, \quad \xi_{i}=\mathfrak{d}_{i}\left(-\ln \|\xi\|_{\mathfrak{o}_{i}}\right) \xi, \quad i=1,2
\end{gathered}
$$

stabilizes the origin of the system (16) in a fixed time $T\left(x_{0}\right) \leq T_{\max }:=\frac{c}{-\mu_{1} \alpha_{1}}+$ $\frac{c}{\mu_{2} \alpha_{2}}, \alpha_{1}=\inf _{\|\xi\|=1} \frac{\partial\|\xi\|}{\partial \xi} G_{\mathfrak{D}_{1}} \xi, \alpha_{2}=\inf _{\|\xi\|=1} \frac{\partial\|\xi\|}{\partial \xi} G_{\mathfrak{D}_{2}} \xi$, and $\|u(t)\|<+\infty$ for $t \geq 0$, $\|u(t)\| \rightarrow 0$ as $t \rightarrow T\left(x_{0}\right)$.

Proof. Let us consider the system $\dot{\xi}=f_{i}(\xi), f_{i}=\left(\begin{array}{c}f \\ g_{i}\end{array}\right), i=1,2$. By construction $f_{i}$ is $\mathfrak{d}_{i}$-homogeneous of degree $\mu_{i}$. From (18) we derive $\frac{\partial\|\xi\|}{\partial \xi} f_{i}(\xi) \leq-c$ for $\|\xi\|=1$. Corollary 9 implies that in this case the homogeneous norm $\|\cdot\|_{\mathfrak{d}_{i}}$ is the Lyapunov function to the corresponding system. The closed loop-system (16), (21) has the

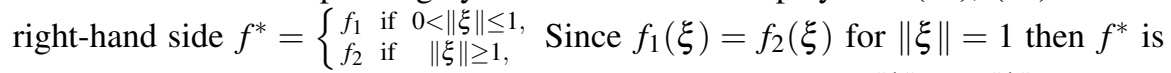

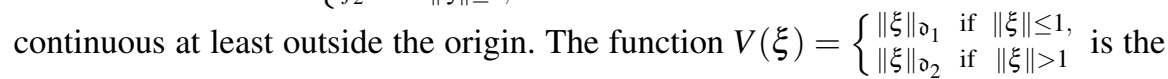
Lyapunov function of the closed-loop system. Indeed, it is continuous at zero, Lipschitz continuous on $\mathbb{R}^{n+m} \backslash\{\boldsymbol{0}\}$ and continuously differentiable on $\mathbb{R}^{n+m} \backslash S \backslash\{\mathbf{0}\}$, where $S$ is a unit sphere in $\mathbb{R}^{n+m}$. Using formula (9) we derive $\dot{V}(z) \leq-\frac{c}{\alpha_{1}} V^{1+\mu_{1}}$ if $0<V<1, \dot{V}(z) \leq-\frac{c}{\alpha_{2}} V^{1+\mu_{2}}$ if $V>1$ and $\dot{V}(z)=\limsup _{h \rightarrow 0^{+}} \frac{V\left(z+h f^{*}\right)-V(z)}{h} \leq$ $-\frac{c}{\max \left\{\alpha_{1}, \alpha_{2}\right\}}$ if $V=1$. Taking into account $\mu_{1}<0$ and $\mu_{2}>0$ we immediately derive fixed-time stability of the closed-loop system.

The similar result can be extended to the output-based control design for affine single input single output system with relative degree $n$.

Theorem 9 (Dynamical Output-Feedback for SISO System $(m=k=1)$ ).

- Let $\overline{\mathfrak{d}}_{1}$ and $\overline{\mathfrak{d}}_{2}$ be dilations in $\mathbb{R}^{n}$.

- Let the vector field $\tilde{f}=\left(\begin{array}{l}f \\ 0\end{array}\right): \mathbb{R}^{n+1} \rightarrow \mathbb{R}^{n}$ be continuous, $\left(\begin{array}{c}\overline{\mathfrak{d}}_{1}(s) \\ e^{q_{1} s}\end{array}\right)$-homogeneous of negative degree $\mu_{1}<0$ and $\left(\begin{array}{c}\overline{\mathfrak{d}}_{2}(s) \\ e^{q_{2} s}\end{array}\right)$-homogeneous of positive degree $\mu_{2}>0$, where $q_{1}>0, q_{2}>0$.

- Let the measurement function $h: \mathbb{R}^{n} \rightarrow \mathbb{R}$ be $\overline{\mathfrak{d}}_{1}$-homogeneous of degree $\kappa_{1}>$ $-(n-1) \mu_{1}$ and $\overline{\mathfrak{d}}_{2}$-homogeneous of degree $\kappa_{2}>0$.

- Let $H:=\left(\begin{array}{c}h \\ L_{f} h \\ (n-1) \\ (n-1)\end{array}\right)$ be independent of $u$ and the map $H: \mathbb{R}^{n} \rightarrow \mathbb{R}^{n}$
be global diffeomorphism in $\mathbb{R}^{n}$.

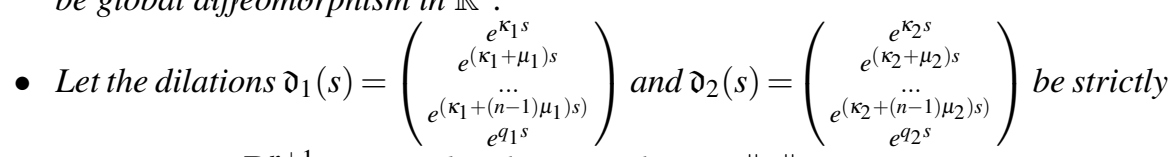
monotone on $\mathbb{R}^{n+1}$ equipped with a smooth norm $\|\cdot\|$. 
If there exist $\gamma>0$ and $c>0$ such that

$$
\frac{\partial\left\|\left(\begin{array}{c}
H(x) \\
u
\end{array}\right)\right\|}{\partial x} f(x, u)+c \leq \gamma \frac{\partial\left\|\left(\begin{array}{c}
H(x) \\
u
\end{array}\right)\right\|}{\partial u}\left[\frac{\partial\left\|\left(\begin{array}{c}
H(x) \\
u
\end{array}\right)\right\|}{\partial u}\right]^{\top} \quad \text { for } \quad\left\|\left(\begin{array}{c}
H(x) \\
u
\end{array}\right)\right\|=1
$$

then the dynamical output-based control

$$
\begin{gathered}
\dot{u}=\left\{\begin{array}{l}
g_{1}(z) \text { if }\|z\| \leq 1, \quad u(0)=0, \quad z=\left(y, \dot{y}, \ldots, y^{(n-1)}, u\right)^{\top}, \\
g_{2}(z) \text { if }\|z\|>1,
\end{array}\right. \\
g_{i}(z)=-\gamma\|z\|_{\mathfrak{d}_{i}}^{q_{i}+\mu_{i}}\left[\left.\frac{\partial\|z\|}{\partial u}\right|_{z=z_{i}}\right]^{\top}, \quad z_{i}=\mathfrak{d}_{i}\left(-\ln \|z\|_{\mathfrak{d}_{i}}\right) z, \quad i=1,2
\end{gathered}
$$

stabilizes the origin of the system (16) in a fixed time $T\left(x_{0}\right) \leq T_{\max }:=\frac{c}{-\mu_{1} \alpha_{1}}+$ $\frac{c}{\mu_{2} \alpha_{2}}, \alpha_{1}=\inf _{\|z\|=1} \frac{\partial\|z\|}{\partial \xi} G_{\mathfrak{d}_{1}} z, \alpha_{2}=\inf _{\|z\|=1} \frac{\partial\|z\|}{\partial \xi} G_{\mathfrak{d}_{2}} z$, and $\|u(t)\|<+\infty$ for $t \geq 0$, $\|u(t)\| \rightarrow 0$ as $t \rightarrow T\left(x_{0}\right)$.

Proof. Let us consider the system $\dot{\xi}=f_{i}(\xi):=\left(\begin{array}{c}f(x, u) \\ g_{i}(H(x), u)\end{array}\right), \xi=\left(\begin{array}{l}x \\ u\end{array}\right), i=1,2$. Taking into account $y^{(j)}=\mathscr{L}_{f}^{(j)} h$ and $\operatorname{deg}_{\mathfrak{d}_{i}}\left(\mathscr{L}_{f}^{(j)} h\right)=\kappa_{i}+j \mu_{i}$ we derive by construction $f_{i}$ is $\left(\begin{array}{c}\overline{\mathfrak{d}}_{i}(s) \\ e^{q_{i} s}\end{array}\right)$-homogeneous of degree $\mu_{i}$. Since the map $H: \mathbb{R}^{n} \rightarrow \mathbb{R}^{n}$ is the global diffeomorphism then the map $\tilde{H}: \mathbb{R}^{n+1} \rightarrow \mathbb{R}^{n+1}$ defines as $\tilde{H}(\xi):=\left(\begin{array}{c}H(x) \\ u\end{array}\right)$ is global diffeomorphism on $\mathbb{R}^{n+1}$. Note that $\kappa_{i}+(n-1) \mu_{i}>0$ implies $\tilde{H}(\mathbf{0})=\mathbf{0}$ and $\tilde{H}(\xi) \neq \mathbf{0}$ if $\xi \neq \mathbf{0}$. Since $\left\|\tilde{H}\left(\left(\begin{array}{c}\overline{\mathfrak{d}}_{i}(s) \\ e^{q_{i} s}\end{array}\right) \xi\right)\right\|_{\mathfrak{d}_{i}}=\left\|\mathfrak{d}_{i}(s) \tilde{H}(\xi)\right\|_{\mathfrak{d}_{i}}=e^{s}\|\tilde{H}(\xi)\|_{\mathfrak{d}_{i}}$ then the function $V_{i}: \mathbb{R}^{n+1} \rightarrow \mathbb{R}_{+}$defined as $V_{i}(\xi)=\|\tilde{H}(\xi)\|_{\mathfrak{d}_{i}}$ is $\left(\begin{array}{c}\overline{\mathfrak{d}}_{i}(s) \\ e^{q_{i} s}\end{array}\right)$-homogeneous of degree 1 , continuous on $\mathbb{R}^{n+1}$, smooth outside the origin (due to strict monotonicity of the dilation $\mathfrak{d}_{i}$ and differentiability of $\tilde{H}$ ) and radially unbounded. From (18) we derive $\frac{\partial\|\tilde{H}(\xi)\|}{\partial \xi} f_{i}(\xi) \leq-c$ for $\|\tilde{H}(\xi)\|=1$. Note that $f_{1}(\xi)=f_{2}(\xi)$ for $\|\xi\|=1$, so the closed loop-system (16), (21) has continuous right-hand side. The function $V(\xi)=\left\{\begin{array}{l}V_{1}(\xi) \text { if }\|\tilde{H}(\xi)\| \leq 1, \\ V_{2}(\xi) \text { if }\|\tilde{H}(\xi)\|>1\end{array}\right.$ is the Lyapunov function for the closed-loop system. This Lyapunov function is Lipschitz continuous on $\mathbb{R}^{n+m}$, continuously differentiable on $\mathbb{R}^{n+1} \backslash \tilde{S} \backslash\{\boldsymbol{0}\}$, where $\tilde{S}=\left\{\xi \in \mathbb{R}^{n+1}:\|\xi\|=1\right\}$ is a unit level set of the function $V$. Using formula (9) we derive $\dot{V}(z) \leq-\frac{c}{\alpha_{1}} V^{1+\mu_{1}}$ if $0<V<1$, $\dot{V}(z) \leq-\frac{c}{\alpha_{2}} V^{1+\mu_{2}}$ if $V>1$ and $\dot{V}(z)=\limsup _{h \rightarrow 0^{+}} \frac{V\left(z+h f^{*}\right)-V(z)}{h} \leq-\frac{c}{\max \left\{\alpha_{1}, \alpha_{2}\right\}}$ if $V=1$. Taking into account $\mu_{1}<0$ and $\mu_{2}>0$ we immediately derive fixed-time stability of the closed-loop system. 


\section{Discussions and Conclusions}

\subsection{Summary of the Obtained Results}

- The notions of fast stability are surveyed. Quantitative characteristics of fast stability (like hyper exponential convergence rate) are introduced.

- The concept of generalized homogeneity is introduced for ODE as a main tool for fast control design.

- Two (state-based and output-based) schemes of dynamical fixed-time control design are proposed for plants described by nonlinear ODE.

\subsection{On Drawback Finite-Time Stability for Time-Delay Systems}

The examples given above present hyper exponential systems with non-asymptotic transitions (solutions vanish in a finite time). However, finite-time convergence to zero is rather rare or sometimes impossible for time delay models. For example, let us consider the scalar time delay system

$$
\dot{x}(t)=f_{0}(x(t))+f_{1}(x(t-h)), \quad t>0,
$$

where $x(t) \in \mathbb{R}, f_{1}, f_{2}: \mathbb{R} \rightarrow \mathbb{R}$ are continuous functions, and $h>0$ is a constant delay. This system has continuously differentiable solutions [21] for any smooth initial conditions

$$
x(\tau)=\psi(\tau), \quad \tau \in[-h, 0], \quad \phi \in C([-h, 0], \mathbb{R}) .
$$

Let us assume that $x=0$ is the equilibrium of the system and $f_{1}$ is non-trivial in any neighbourhood of $x=0$, i.e. for $\forall \varepsilon>0, \exists x \in \mathbb{R}:|x|<\varepsilon$ such that $f_{1}(x) \neq 0$. If we omit the latter assumption then the considered time-delay system degenerates to delay-free one in some neighbourhood of the origin.

We claim that the zero solution of the time-delay system is never finite-time stable. In order words there is no continuous function $f_{0}$ such that the considered system is finite-time sable. Indeed, let us suppose the contrary, i.e.

$$
\exists T>0, \exists \delta>0 \quad \text { such that } \quad x(t) \neq 0, t \in(T-\delta, T) \quad \text { and } \quad x(t)=0, t>T .
$$

In this case, $f_{0}(x(t))=0$ and $\dot{x}(t)=f_{1}(x(t-h))$ for $t>T$. Since $x(t) \neq 0$ for $t \in(T-$ $\delta, T)$ then $\dot{x}(t)$ is not identically zero for some $t>T$. This together with continuous differentiability of $x$ implies the contradiction to $x(t)=0$ for $t>T$.

Therefore, ideas of non-asymptotic (finite-time/fixed-time) stabilization are inconsistent with some time delay systems (see also [16] for more details). However, they may demonstrate asymptotic hyper exponential transitions [38]. 


\subsection{Fast Transition of PDEs}

\subsubsection{Fixed-time extinction of waves}

Hyper exponential transitions also appears in distributed parameters systems. Indeed, let us consider the wave equation

$$
u_{t t}=u_{x x}, \quad t>0, \quad x \in[0,1], \quad u: \mathbb{R}_{+} \times[0,1] \rightarrow \mathbb{R},
$$

with the so-called "transparent" boundary condition

$$
u_{x}(t, 0)=u_{t}(t, 0), \quad u_{x}(t, 1)=-u_{t}(t, 1)
$$

and the initial conditions

$$
u(0, x)=\phi(x), \quad u_{t}(0, x)=\psi
$$

from $\left\{(\phi, \psi) \in H^{1}((0,1), \mathbb{R}) \times L^{2}((0,1), \mathbb{R}): \phi(0)+\phi(1)+\int_{0}^{1} \psi(s) d s=0\right\}$, where $L^{2}$ and $H^{1}$ are Lebesgue and Sobolev spaces, respectively. The boundary conditions are transparent in the sense that the wave $u(t, x)=f(x-t)$ traveling to the right leave the domain at $x=1$ without generating any reflected wave and the wave $u(t, x)=$ $f(x+t)$ traveling to the right leave the domain at $x=0$ similarly.

It is well-known (see, e.g. [31]) that solution of such wave equation vanishes for $t \geq 1$ independently of the initial condition. In the context of distributed parameters systems this effect is known as finite-time extinction. Obviously, the norm of $u$ tends to zero hyper exponentially with respect to time variable $t$.

This example shows that in the case of distributed parameters systems the hyper exponential and finite-time/fixed-time transitions can be observed in linear models (see also [5], [13]).

\subsubsection{Generalized Homogeneity of Infinite Dimensional Systems}

The extension of the homogeneity concept to infinite dimensional systems has been presented in [35], where a lot of important properties of homogeneous dilations, functional and operators have been discovered in the case of Banach spaces. The developed homogeneous framework looks promising for extension of the existing results on fast control and estimation to PDEs and Time-Daly Systems.

\section{Acknowledgments}

This study is partially supported by The French National Research Agency, Grant ANR Finite4SoS (ANR 15 CE23 0007) and the Russian Federation Ministry of Education and Science, contract/grant numbers 02.G25.31.0111 and 14.Z50.31.0031. 


\section{References}

1. Adamy, J., Flemming, A.: Soft variable-structure controls: a survey. Automatica 40, 18211844 (2004)

2. Andrieu, V., Praly, L., Astolfi, A.: Homogeneous Approximation, Recursive Observer Design, and Output Feedback. SIAM Journal of Control and Optimization 47(4), 1814-1850 (2008)

3. Armstrong-Helouvry, B.: Control of machines with friction. Springer (1991)

4. Bacciotti, A., Rosier, L.: Lyapunov Functions and Stability in Control Theory. Springer (2001)

5. Balakrishnan, A.: Superstability of systems. Applied Mathematics and Computation 164, 321-326 (2005)

6. Bhat, S., Bernstein, D.: Finite time stability of continuous autonomous systems. SIAM J. Control Optim. 38(3), 751-766 (2000)

7. Bhat, S.P., Bernstein, D.S.: Geometric homogeneity with applications to finite-time stability. Mathematics of Control, Signals and Systems 17, 101-127 (2005)

8. Clarke, F.: Optimization and Nonsmooth Analysis. SIAM, Philadelphia (1990)

9. Clarke, F.H., Ledyaev, Y.S., Stern, R.: Asymptotic stability and smooth Lyapunov functions. Journal of differential Equations 149, 69-114 (1998)

10. Coron, J.M., Nguyen, H.M.: Null controllability and finite time stabilization for the heat equations with variable coefficients in space in one dimension via backstepping approach. (https://hal.archives-ouvertes.fr/hal-01228895) (2015)

11. Coron, J.M., Praly, L.: Adding an integrator for the stabilization problem. Systems \& Control Letters 17(2), 89-104 (1991)

12. Courant, R., John, F.: Introduction to calculus and analysis (Vol. II/1). New York: Springer (2000)

13. Creutz, D., Mazo, M.J., Preda, C.: Superstability and finite time extinction for $c_{0}$-semigroups. arXiv:0907.4812v4 (2013)

14. Cruz-Zavala, E., Moreno, J., Fridman, L.: Uniform robust exact differentiator. IEEE Transactions on Automatic Control 56(11), 2727-2733 (2011)

15. Efimov, D., Perruquetti, W.: Oscillations conditions in homogenous systems. In: Proc. IFAC NOLCOS Symp., pp. 1379-1384 (2010)

16. Efimov, D., Polyakov, A., Fridman, E., Perruquetti, W., Richard, J.P.: Comments on finite-time stability of time-delay systems. Automatica 50(7), 1944-1947 (2014)

17. Falkovich, G.: Fluid Mechanics (A short course for physicists). Cambridge University Press. (2011)

18. Filippov, A.F.: Differential Equations with Discontinuous Righthand Sides. Kluwer Academic Publishers (1988)

19. Galaktionov, V.A., Vazquez, J.L.: Necessary and sufficient conditions for complete blow-up and extinction for one-dimensional quasilinear heat equations. Archive for Rational Mechanics and Analysis 129(3), 225-244 (1995)

20. Haimo, V.: Finite time controllers. SIAM Journal of Control and Optimization 24(4), 760-770 (1986)

21. Hale, J.: Theory of Functional Differential Equations. Springer-Verlag (1977)

22. Hermes, H.: Nilpotent approximations of control systems and distributions. SIAM Journal of Control and Optimization 24(4), 731 (1986)

23. Kawski, M.: Geometric homogeneity and stabilization. In: A. Krener, D. Mayne (eds.) Proc. IFAC Nonlinear Control Symposium, pp. 164-169. Lake Tahoe, CA (1995)

24. Khomenuk, V.V.: On systems of ordinary differential equations with generalized homogenous right-hand sides. Izvestia vuzov. Mathematica. 3(22), 157-164 (1961 (in Russian))

25. Korobov, V.: A general approach to synthesis problem. Doklady Academii Nauk SSSR 248, 1051-1063 (1979)

26. Levant, A.: Homogeneity approach to high-order sliding mode design. Automatica 41(5), 823-830 (2005)

27. Lyapunov, A.M.: The general problem of the stability of motion. Taylor \& Francis (1992) 
28. Moulay, E., Perruquetti, W.: Finite-time stability of differential inclusions. IMA Journal Mathematical Control and Information Sciences 22(4), 465-475 (2005)

29. Orlov, Y.: Finite time stability and robust control synthesis of uncertain switched systems. SIAM Journal of Control and Optimization 43(4), 1253-1271 (2005)

30. Pazy, A.: Semigroups of Linear Operators and Applications to Partial Differential Equations. Springer (1983)

31. Perrollaz, V., Rosier, L.: Finite-time stabilization of $2 \times 2$ hyperbolic systems on tree-shaped networks. SIAM Journal of Control and Optimization 52(1), 143-163 (2014)

32. Perruquetti, W., Floquet, T., Moulay, E.: Finite-time observers: application to secure communication. IEEE Transactions on Automatic Control 53(1), 356-360 (2008)

33. Polyakov, A.: Nonlinear feedback design for fixed-time stabilization of linear control systems. IEEE Transactions on Automatic Control 57(8), 2106-2110 (2012)

34. Polyakov, A., Coron, J.M., Rosier, L.: On finite-time stabilization of evolution equations: A homogeneous approach. In: Conference on Decision and Control, pp. (https://hal.inria.fr/hal01371,089) (2016)

35. Polyakov, A., Efimov, D., Fridman, E., Perruquetti, W.: On homogeneous distributed parameters equations. IEEE Transactions on Automatic Control 61(11), 3657-3662 (2016)

36. Polyakov, A., Efimov, D., Perruquetti, W.: Finite-time and Fixed-time Stabilization: Implicit Lyapunov Function Approach. Automatica 51(1), 332-340 (2015)

37. Polyakov, A., Efimov, D., Perruquetti, W.: Robust stabilization of mimo systems in finite/fixed time. International Journal of Robust and Nonlinear Control 26(1), 69-90 (2016)

38. Polyakov, A., Efimov, D., Perruquetti, W., Richard, J.P.: Implicit Lyapunov-Krasovski Functionals for Stability Analysis and Control Design of Time-Delay Systems. IEEE Transactions on Automatic Control 60(12), 3344-3349 (2015)

39. Polyakov, A., Fridman, L.: Stability notions and lyapunov functions for sliding mode control systems. ournal of The Franklin Institute 351(4), 1831-1865 (2014)

40. Poznyak, A.: Advanced Mathematical Tools for Automatic Control Engineers. Volume 1: Deterministic Technique. Elsevier (2008)

41. Rosier, L.: Homogeneous Lyapunov function for homogeneous continuous vector field. Systems \& Control Letters 19, 467-473 (1992)

42. Rosier, L.: Etude de quelques problemes de stabilization. PhD Thesis, Ecole Normale Superieure de Cachan (France) (1993)

43. Roxin, E.: On finite stability in control systems. Rendiconti del Circolo Matematico di Palermo 15, 273-283 (1966)

44. Roxin, E.: On stability in control systems. J. SIAM Control 3, 357-372 (1966)

45. Ruzhansky, M., Sugimoto, M.: On global inversion of homogeneous maps. Bull. Math. Sci. 5, 13-18 (2015)

46. Sabinina, E.: A class of non-linear degenerating parabolic equations. Soviet Methematics Doklady 148, 495-498 (1962)

47. Tikhonov, A.: Systems of differential equations containing a small parameter multiplying the derivative (in russian). Math. Sbornik 31(73), 575-586 (1952)

48. Utkin, V.I.: Sliding Modes in Control Optimization. Springer-Verlag, Berlin (1992)

49. Zubov, V.: On systems of ordinary differential equations with generalized homogenous righthand sides. Izvestia vuzov. Mathematica. 1, 80-88 (1958 (in Russian))

50. Zubov, V.I.: Methods of A.M. Lyapunov and Their Applications. Noordhoff, Leiden (1964) 